\title{
Justifying Social Discounting: The Rank-Discounted Utilitarian Approach
}

\author{
Stéphane Zuber \\ Geir B. Asheim
}

CESIFO WORKING PAPER No. 3192

CAtegory 2: Public CHOICE

SEPTEMBER 2010

\footnotetext{
An electronic version of the paper may be downloaded

- from the SSRN website:

- from the RePEc website:

- from the CESifo website:

www.SSRN.com

Www.RePEc.org

www.CESifo-group.org/wp
} 


\title{
Justifying Social Discounting: The Rank-Discounted Utilitarian Approach
}

\begin{abstract}
The discounted utilitarian criterion for infinite horizon social choice has been criticized for treating generations unequally. We propose an extended rank-discounted utilitarian (ERDU) criterion instead. The criterion amounts to discounted utilitarianism on non-decreasing streams, but it treats all generations impartially: discounting becomes the mere expression of intergenerational inequality aversion. We show that more inequality averse ERDU societies have higher social discount rates when future generations are better-off. We apply the ERDU approach in two benchmark economic growth models and prove that it promotes sustainable policies that maximize discounted utilitarian welfare.
\end{abstract}

JEL-Code: D63, H43, Q56.

Keywords: intergenerational equity, social discounting, discounted utilitarianism, sustainability.

\author{
Stéphane Zuber \\ CORE \\ Catholic University of Louvain \\ Voie du Roman Pays 34 \\ 1348 Louvain-La-Neuve \\ Belgium \\ Stephane.Zuber@uclouvain.be
}

\author{
Geir B. Asheim \\ Department of Economics \\ University of Oslo \\ P.O. Box 1095 Blindern \\ 0317 Oslo \\ Norway \\ g.b.asheim@econ.uio.no
}

September 26, 2010

We thank Antoine Bommier, Wolfgang Buchholz, Christian Gollier, Luc Lauwers, Michel Le Breton, John Roemer, an anonymous referee and seminar audiences in Berlin, Louvain, Montréal, Moscow, Oslo, Paris and Toulouse for their comments and useful references. Zuber thanks in particular Marc Fleurbaey and François Maniquet for provocative and fruitful discussions at an early stage of this project. This paper is part of the research activities at the center of Equality, Social Organization, and Performance (ESOP) at the Department of Economics at the University of Oslo. ESOP is supported by the Research Council of Norway. 


\section{Introduction}

The most popular objective function used to determine optimal policies in infinite horizon models is the discounted utilitarian criterion,

$$
\sum_{t \in \mathbb{N}} \beta^{t-1} u\left(x_{t}\right)
$$

where $0<\beta<1$ is the social discount factor and $x_{t}$ is the consumption of generation $t$. This criterion has been heavily criticized on the ground that it treats successive generations differently. Many economists in the utilitarian tradition have denounced this deviation from the ideal of equal treatment of all individuals. For instance, Frank Ramsey famously described discounting as a "practice which is ethically indefensible and arises merely from the weakness of the imagination" (Ramsey, 1928, p. 543). Among others, Pigou (1920) and Harrod (1948) have also stigmatized discounting.

Drawing on these criticisms, a prolific literature has studied whether it would be possible to combine the principle of procedural equity (equal treatment of all generations) with the Pareto principle in the context of infinite consumption streams. Although some positive results have been obtained, most of this literature stemming from Diamond (1965) has reached negative conclusions (Basu and Mitra, 2003; Zame, 2007; Lauwers, 2010).

At the same time, several authors have pointed out the distributional consequences of not discounting future generations' utility. Mirrlees (1967) computed optimal intertemporal consumption patterns in plausible economic models using the undiscounted utilitarian criterion (the so-called Ramsey criterion). He observed that present generations should save up to $50 \%$ of their net income for the sake of future generations. The finding was best summarized by philosopher John Rawls who declared that "the utilitarian doctrine may direct us to demand heavy sacrifices of the poorer generations for the sake of greater advantages for the later ones that are far better off" (Rawls, 1999, p. 253). He went on saying that "these consequences can be to some degree corrected by discounting the welfare of those living in the future" (Rawls, 1999, p. 262).

Although Rawls did not endorse discounted utilitarianism (for the very reason that its failure to comply with procedural equity "has no intrinsic ethical appeal" Rawls, 1999, p. 262), most of the economic literature has adopted it, as the lesser of two evils. Yet the conflict between procedural equity and distributional equity in a utilitarian context has remained unsolved.

The above distributional justification for discounted utilitarianism critically relies on the assumption that future generations are better off in the implemented intergenerational allocation. Asheim and Buchholz (2003) noticed that, in certain technological contexts, for instance in the Dasgupta-Heal-Solow model 
of capital accumulation and resource depletion, future generations may not be better off. Undiscounted utilitarianism may then yield more satisfactory recommendations than discounted utilitarianism. The key point is that, for discounting to prevent high sacrifices for the sake of others that are better off, it is critical that generations' rank in time corresponds to their rank in well-being.

If we retain the interpretation of the discount factor as preventing high sacrifices from the poor, it looks closely related to the social weights used in rankdependent measures of social welfare. An example of a rank-dependent criterion is the Gini social welfare function. Generalizations thereof have been proposed by Weymark (1981) and Ebert (1988). The main feature of rank-depended social welfare functions is that they put more weight on the utility of the worse off. Rank-dependent weights simply represent the society's aversion to inequality.

In this paper, we propose to apply rank-dependent methods to intergenerational justice. ${ }^{1}$ More precisely, we put forward that the social observer use an element from the class of rank-discounted utilitarian social welfare functions:

$$
\sum_{r \in \mathbb{N}} \beta^{r-1} u\left(x_{[r]}\right)
$$

Here, the consumption stream $\left(x_{[1]}, x_{[2]}, \ldots, x_{[r]}, \ldots\right)$ is a reordering of the consumption stream $\left(x_{1}, x_{2}, \ldots, x_{t}, \ldots\right)$ such that $x_{[1]} \leq x_{[2]} \leq \cdots \leq x_{[r]} \leq \cdots$.

However, an obstacle to applying rank-discounted utilitarianism in the context of infinite consumption streams is that consumption streams where some generations have infinite ranks cannot be reordered into a non-decreasing stream. We resolve this problem by showing how the rank-discounted utilitarian approach can be extended in a natural manner to the full domain by including also consumption streams that cannot be reordered into non-decreasing streams.

The extended rank-discounted utilitarian approach coincides with discounted utilitarianism on the set of non-decreasing consumption streams. Utility discounting is then justified as an expression of inequality aversion when future generations are better off. However, and contrary to the discounted utilitarian approach, extended rank-discounted utilitarianism also satisfies procedural equity: two intergenerational consumption streams that are identical up to a permutation are deemed equally good. Furthermore, it satisfies the strong Pareto principle on the the domain of streams that can be reordered into non-decreasing streams. Hence, the extended rank-discounted utilitarian approach overcomes the impossibility results in the tradition of Diamond (1965) on this domain.

In Section 3, we offer a complete characterization of extended rank-discounted utilitarian preferences. This characterization is clearly related to Koopmans'

\footnotetext{
${ }^{1}$ Alternatively, the analysis could have been motivated as an extension of known results on finite rank-dependent social evaluation to the infinite case. For a comparison of our analysis with relevant contributions in this alternative setting, see subsection 3.2.
} 
(1960) characterization of discounted utilitarian preferences. The difference is that his separability and stationarity axioms are imposed on non-decreasing streams only. Separability axioms on ordered streams are common in the theory of decision under uncertainty (Gilboa, 1987; Wakker, 1993) and in the theory of inequality measurement (Weymark, 1981; Ebert, 1988). With the exception of Rébillé (2007), they have never been used in the theory of intertemporal decision making yet. They permit to weight utilities according to their rank in a distribution, which is exactly what rank-discounted utilitarian criteria do.

In Section 4, we provide conditions for a social observer using an extended rank-discounted utilitarian criterion to be inequality averse, in the sense that she always prefers a consumption stream obtained from another through a PigouDalton redistributive transfer. We also provide conditions for comparing two social observers in terms of inequality aversion. When the social observer has homothetic preferences, these conditions are very simple: she needs to discount ranks more and to use a more concave utility function.

Distributional equity in the spirit of Atkinson (1970) has been addressed in many papers in the literature on intergenerational equity (see, e.g., Birchenhall and Grout, 1979; Bossert, Sprumont and Suzumura, 2007; Hara, Shinotsuka, Suzumura and $\mathrm{Xu}, 2008)$. However, this literature did not emphasize the effects of inequality aversion on society's choice. We claim that inequality aversion is a central notion for evaluating intergenerational problems.

In Section 5, we explore the implications of rank-discounted utilitarian social welfare functions for the social discount rate. The highly publicized debates on the social discount rate in the context of climate change have highlighted its importance for policy evaluation. An 'ethical' view has suggested low values for the social discount rate, on the ground that pure-time discounting violates procedural equity. Rank-discounted utilitarianism suggests an alternative 'ethical' view where discounting is an expression of society's aversion to inequality.

Indeed, we prove that a more inequality averse social observer always discount the future more, provided that future generations are better off. This has important policy implications. If future generations are expected to be better off in spite of climate change, then a more inequality averse extended rankdiscounted utilitarian social observer will agree with the recommendation of Nordhaus (2008) to have gradual emission control policies rather than that of Stern (2006) who calls for immediate action. However, since rank-discounting depends on a generation's rank in the intergenerational distribution rather than its rank in time, if future generations are expected to be less well-off because of climate change, then the social discount rate should on the contrary be negative, and strong action should be undertaken to mitigate climate change.

In Section 6, we show that the extended rank-discounted utilitarian approach can be applied to find the optimal growth policy in two benchmark models: the 
Ramsey growth model and the Dasgupta-Heal-Solow model of capital accumulation and resource depletion. Also in these applications, inequality aversion plays a crucial role. Indeed, in a more inequality averse society, growth is prevented for a greater set of initial conditions: if the initial stock of capital is high enough, the society prefers to maintain consumption forever. Then more inequality aversion yields greater equality and lower long-run consumption.

To reach these conclusions, we start in Section 2 by introducing the framework of our analysis.

\section{The framework}

Let $\mathbb{N}$ denote as usual the set of natural numbers $\{1,2,3, \ldots\}$. Let $\mathbb{R}$ denote the set of real numbers, $\mathbb{R}_{+}$the set of nonnegative real numbers, and $\mathbb{R}_{++}$the set of positive real numbers.

Denote by $\mathbf{x}=\left(x_{1}, x_{2}, \ldots, x_{t}, \ldots\right)$ an infinite stream (or allocation), where $x_{t} \in \mathbb{R}_{+}$is a one-dimensional indicator of the well-being of generation $t$. We refer to this indicator as the consumption of generation $t$, restrict attention to allocations consisting of bounded consumption streams, and denote by

$$
\mathbf{X}=\left\{\mathbf{x}=\left(x_{1}, \ldots, x_{t}, \ldots\right) \in \mathbb{R}_{+}^{\mathbb{N}}: \sup _{t} x_{t}<+\infty\right\}
$$

the set of possible allocations.

For $\mathbf{x}, \mathbf{y} \in \mathbf{X}$, write $\mathbf{x} \geq \mathbf{y}$ whenever $x_{t} \geq y_{t}$ for all $t \in \mathbb{N}$; write $\mathbf{x}>\mathbf{y}$ if $\mathbf{x} \geq \mathbf{y}$ and $\mathbf{x} \neq \mathbf{y}$; and write $\mathbf{x} \gg \mathbf{y}$ whenever $x_{t}>y_{t}$ for all $t \in \mathbb{N}$. For any $T \in \mathbb{N}$ and $\mathbf{x}, \mathbf{y} \in \mathbf{X}$, denote by $\mathbf{x}_{\mathbf{T}} \mathbf{y}$ the consumption stream $\mathbf{z}$ such that $z_{t}=x_{t}$ for all $t \leq T$ and $z_{t}=y_{t}$ for all $t>T$. For any $x \in \mathbb{R}_{+}$and $\mathbf{y} \in \mathbf{X}$, denote by $(x, \mathbf{y})$ the stream $\left(x, y_{1}, y_{2}, \ldots\right)$.

Three subsets of $\mathbf{X}$ will be of particular interest. First, we introduce the set of stationary consumption streams, $\mathbf{X}^{\mathbf{c}}=\left\{\mathbf{x}^{\mathbf{c}}, x \in \mathbb{R}_{+}\right\}$, where for any $x \in \mathbb{R}_{+}$, $\mathbf{x}^{\mathbf{c}} \in \mathbf{X}$ denotes the allocation such that $x_{t}^{c}=x$ for all $t \in \mathbb{N}$.

A second subset of $\mathbf{X}$ is the set of non-decreasing streams in $\mathbf{X}$. This set is denoted $\mathbf{X}^{+}=\left\{\mathbf{x} \in \mathbf{X}: x_{t} \leq x_{t+1}, \forall t \in \mathbb{N}\right\}$.

The third subset of $\mathbf{X}$, playing a key role in the remainder of the paper, is the set of allocations, $\overline{\mathbf{X}}$, whose elements can be permuted to obtain non-decreasing streams. To introduce $\overline{\mathbf{X}}$ formally, let $\Pi$ be the set of all permutations on $\mathbb{N}$. For any $\pi \in \Pi$ and $\mathbf{x} \in \mathbf{X}$, let $\mathbf{x}_{\pi}=\left(x_{\pi(1)}, x_{\pi(2)}, \ldots, x_{\pi(t)}, \ldots\right)$. The set $\overline{\mathbf{X}}$ is defined as follows: $\overline{\mathbf{X}}=\left\{\mathbf{x} \in \mathbf{X}: \exists \pi \in \Pi, \mathbf{x}_{\pi} \in \mathbf{X}^{+}\right\}$.

The following inclusions hold: $\mathbf{X}^{\mathbf{c}} \subset \mathbf{X}^{+} \subset \overline{\mathbf{X}} \subset \mathbf{X}$. In a finite setting, $\overline{\mathbf{X}}$ would be the same as $\mathbf{X}$. To see why this does not hold in an infinite setting, consider the stream $\mathbf{x}=(1,0,0, \ldots)$. For any $\pi \in \Pi$, it must be that $\pi(1)<+\infty$ so that any reordered stream has the form $(0,0, \ldots, 0,1,0, \ldots)$. Hence, $\mathbf{x}$ cannot 
be reordered to form a non-decreasing stream.

To characterize the set $\overline{\mathbf{X}}$, let $\ell(\mathbf{x})$ denote $\liminf _{t \rightarrow+\infty} x_{t}$ for any $\mathbf{x} \in \mathbf{X}$. Because streams in $\mathbf{X}$ are bounded, $\ell(\mathbf{x})$ is well-defined for all $\mathbf{x} \in \mathbf{X}$. Write $L(\mathbf{x})=\left\{t \in \mathbb{N}: x_{t}<\ell(\mathbf{x})\right\}$ and denote by $|L(\mathbf{x})|$ the cardinality of $L(\mathbf{x})$.

\section{Proposition 1.}

(a) If an allocation $\mathbf{x} \in \mathbf{X}$ satisfies $|L(\mathbf{x})|<+\infty$, then $\mathbf{x}$ belongs to $\overline{\mathbf{X}}$ if and only if $x_{t} \leq \ell(\mathbf{x})$ for all $t \in \mathbb{N}$.

(b) If an allocation $\mathbf{x} \in \mathbf{X}$ satisfies $|L(\mathbf{x})|=+\infty$, then $\mathbf{x}$ belongs to $\overline{\mathbf{X}}$ if and only if $x_{t}<\ell(\mathbf{x})$ for all $t \in \mathbb{N}$.

Proposition 1 is clearly equivalent to the following lemma.

Lemma 1. An allocation $\mathbf{x} \in \mathbf{X}$ belongs to $\overline{\mathbf{X}}$ if and only if the cardinality of $\left\{t \in \mathbb{N}, t>\tau: x_{t}<x_{\tau}\right\}$ is finite for all $\tau \in \mathbb{N}$.

Proof. For any $\mathbf{x} \in \mathbf{X}$ and $\tau \in \mathbb{N}$, write $\Lambda_{\tau}(\mathbf{x})=\left\{t \in \mathbb{N}, t>\tau: x_{t}<x_{\tau}\right\}$.

If $\left|\Lambda_{\tau}(\mathbf{x})\right|=+\infty$ for some $\tau \in \mathbb{N}$, then, for any $\pi \in \Pi, \pi(\tau)<+\infty$ and it is impossible that $\pi(t)<\pi(\tau)$ for all $t \in \Lambda_{\tau}(\mathbf{x})$. Hence, $\mathbf{x} \notin \overline{\mathbf{X}}$.

Conversely, assume $\left|\Lambda_{\tau}(\mathbf{x})\right|<+\infty$ for all $\tau \in \mathbb{N}$. The set $\Lambda_{1}(\mathbf{x})$ is finite and can be re-ordered in non-decreasing order. These coordinate will form the $n_{1}$ first elements of the ordered stream, with $n_{1}=\left|\Lambda_{1}(\mathbf{x})\right|$. And $\pi(1)=n_{1}+1$. Then let $\tau_{2}$ be the first period such that $x_{\tau_{2}} \geq x_{1}$. The set $\Lambda_{\tau_{2}}(\mathbf{x}) \backslash \Lambda_{1}(\mathbf{x})$ is finite and can be ordered in increasing order. These will form the $n_{2}$ next elements in the ordered stream, with $n_{2}=\left|\Lambda_{\tau_{2}}(\mathbf{x})\right|-\left|\Lambda_{1}(\mathbf{x})\right|$. And $\pi\left(t_{2}\right)=n_{1}+n_{2}+2$. Pursuing this procedure leads to an ordered stream. Hence, $\mathbf{x} \in \overline{\mathbf{X}}$.

For $\mathbf{x} \in \overline{\mathbf{X}}$, denote by $\mathbf{x}_{[]}=\left(x_{[1]}, x_{[2]}, \ldots, x_{[r]}, \ldots\right)$ the non-decreasing allocation which is a permutation of $\mathbf{x}$; i.e., for some $\pi \in \Pi$ such that $\mathbf{x}_{\pi} \in \mathbf{X}_{+}$, it holds that $x_{[r]}=x_{\pi(r)}$ for all $r \in \mathbb{N}$. Note that the permutation $\pi$ need not be unique (if, for instance, $x_{t}=x_{t^{\prime}}$ for some $t \neq t^{\prime}$ ), but the resulting non-decreasing allocation $\mathbf{x}_{[]}$is unique. Likewise, for $\mathbf{x} \in \mathbf{X}$, denote by $\left(x_{[1]}, \ldots, x_{[|L(\mathbf{x})|]}\right)$ the non-decreasing allocation which is a permutation of the elements of $\mathbf{x}$ satisfying $t \in L(\mathbf{x})$. The following notation is useful: $r_{\tau}(\mathbf{x})=\left|\left\{t \in \mathbb{N}: x_{t}<x_{\tau}\right\}\right|+1$ and $\bar{r}_{\tau}(\mathbf{x})=\left|\left\{t \in \mathbb{N}: x_{t} \leq x_{\tau}\right\}\right|$. Whenever $r_{\tau}(\mathbf{x})=\bar{r}_{\tau}(\mathbf{x})<+\infty, r_{\tau}(\mathbf{x})$ is the unique rank of generation $\tau$ in the distribution $\mathbf{x}$, and $x_{\left[r_{\tau}(\mathbf{x})\right]}=x_{\tau}$.

A social welfare relation (SWR) on a set $\mathbf{X}$ is a binary relation $\succsim$, where for any $\mathbf{x}, \mathbf{y} \in \mathbf{X}, \mathbf{x} \succsim \mathbf{y}$ entails that the consumption stream $\mathbf{x}$ is deemed socially at least as good as $\mathbf{y}$. Let $\sim$ and $\succ$ denote the symmetric and asymmetric parts of $\succsim$. A social welfare function (SWF) representing $\succsim$ is a mapping $W: \mathbf{X} \rightarrow \mathbb{R}$ with the property that for any $\mathbf{x}, \mathbf{y} \in \mathbf{X}, W(\mathbf{x}) \geq W(\mathbf{y})$ if and only if $\mathbf{x} \succsim \mathbf{y}$. 


\section{Axiomatic foundation}

The difficulty of combining equal treatment of an infinite number of generations

with sensitivity to the interest of each of these generations has been the topic of a prolific literature since the seminal contribution by Diamond (1965). Although complete social preferences over infinite streams that combine equal treatment with Paretian sensitivity exist (Svensson, 1980), they cannot be represented (Basu and Mitra, 2003) nor explicitly described (Zame, 2007; Lauwers, 2010).

In this section we show how the set of ordered streams serves to overcome this impossibility. In Subsection 3.1 we first impose axioms sufficient to ensure numerical representability. Then we impose Paretian, separability and stationarity axioms, as used to characterize discounted utilitarianism (Koopmans, 1960), but restricted to the set of non-decreasing streams. In Subsection 3.2 we show how this allows us to invoke a strong axiom of equal treatment, requiring social indifference not only for finite permutations (as considered in the literature in the wake of Diamond, 1965), but also for infinite permutations. In the concluding Subsection 3.3 we show that we are still able (i) to retain sensitivity to the interest of any one generation as long as there is only a finite number of other generations with lower consumption levels, and (ii) to satisfy other ethical axioms proposed in the literature to protect the interests of future generations.

\subsection{Axioms}

We first consider axioms sufficient to ensure numerical representability.

Axiom $\mathbf{O}($ Order $)$ The relation $\succsim$ is complete, reflexive and transitive on $\mathbf{X}$.

An SWR satisfying axiom $\mathbf{O}$ is named a social welfare order (SWO).

Axiom $\mathbf{C}$ (Continuity) For any $\mathbf{x}, \mathbf{y} \in \mathbf{X}$, if a sequence $\mathbf{x}^{1}, \mathbf{x}^{2}, \ldots, \mathbf{x}^{k}, \ldots$ of allocations in $\mathbf{X}$ is such that $\lim _{k \rightarrow \infty} \sup _{t \in \mathbb{N}}\left|x_{t}^{k}-x_{t}\right|=0$ and, for all $k \in \mathbb{N}$, $\mathbf{x}^{k} \succsim \mathbf{y}\left(\operatorname{resp} . \mathbf{x}^{k} \precsim \mathbf{y}\right)$, then $\mathbf{x} \succsim \mathbf{y}(\operatorname{resp} . \mathbf{x} \precsim \mathbf{y})$.

Axiom $\mathbf{M}$ (Monotonicity) For any $\mathbf{x}, \mathbf{y} \in \mathbf{X}$, if $\mathbf{x}>\mathbf{y}$, then $\mathbf{x} \succsim \mathbf{y}$.

Axiom $\mathbf{M}$ is implied by the strong Pareto principle.

We then consider an axiom ensuring some sensitivity to the interests of the present generation.

Axiom RD (Restricted Dominance) For any $x, y \in \mathbb{R}_{+}$, if $x>y$, then $\left(x, \mathbf{x}^{\mathbf{c}}\right) \succ$ $\left(y, \mathbf{x}^{\mathbf{c}}\right)$.

Axiom RD is implied by the strong Pareto principle restricted to the set of streams that can be reordered into non-decreasing streams:

Axiom RSP (Restricted Strong Pareto) For any $\mathbf{x}, \mathbf{y} \in \overline{\mathbf{X}}$, if $\mathbf{x}>\mathbf{y}$, then $\mathbf{x} \succ \mathbf{y}$. 
We now turn to restricted versions of the separability and stationarity axioms usually invoked to characterize discounted utilitarianism.

Axiom RSEP (Restricted Separable Present) For any $\mathbf{x}, \mathbf{y}, \mathbf{x}^{\prime}, \mathbf{y}^{\prime} \in \mathbf{X}^{+}$such that (i) $x_{t}=x_{t}^{\prime}$ and $y_{t}=y_{t}^{\prime}$ for all $t \in\{1,2\}$ and (ii) $x_{t}=y_{t}$ and $x_{t}^{\prime}=y_{t}^{\prime}$ for all $t \in \mathbb{N} \backslash\{1,2\}, \mathbf{x} \succsim \mathbf{y}$ if and only if $\mathbf{x}^{\prime} \succsim \mathbf{y}^{\prime}$.

Axiom RSEP is Postulate $3^{\prime}$ a in Koopmans' (1960) characterization of discounted utilitarianism restricted to the set of non-decreasing streams. We suggest that such a restriction might be supported by ethical intuition. In particular, one might accept that the stream $(1,4,5,5,5, \ldots)$ is socially better than $(2,2,5,5,5, \ldots)$, while not accepting that $(1,4,2,2,2, \ldots)$ is socially better than $(2,2,2,2,2, \ldots)$. It is not obvious that we should treat the conflict between the worst-off and the second worst-off generation presented by the first comparison in the same manner as we treat the conflict between the worst-off and the best-off generation put forward by the second comparison.

Axiom RSEP follows from the following axiom by setting $\mathcal{T}=\{1,2\}$.

Axiom RSE (Restricted Separability) For any $\mathbf{x}, \mathbf{y}, \mathbf{x}^{\prime}, \mathbf{y}^{\prime} \in \mathbf{X}^{+}$and any $\mathcal{T} \subset \mathbb{N}$ such that (i) $x_{t}=x_{t}^{\prime}$ and $y_{t}=y_{t}^{\prime}$ for all $t \in \mathcal{T}$ and (ii) $x_{t}=y_{t}$ and $x_{t}^{\prime}=y_{t}^{\prime}$ for all $t \in \mathbb{N} \backslash \mathcal{T}, \mathbf{x} \succsim \mathbf{y}$ if and only if $\mathbf{x}^{\prime} \succsim \mathbf{y}^{\prime}$.

Axiom RSE is closely related to the comonotonic sure-thing principle that has been introduced in the theory of decision under uncertainty (see Gilboa, 1987; Wakker, 1993).

Axiom RSEF (Restricted Separable Future) For any $\mathbf{x}, \mathbf{y}, \mathbf{x}^{\prime}, \mathbf{y}^{\prime} \in \mathbf{X}^{+}$such that (i) $x_{t}=x_{t}^{\prime}$ and $y_{t}=y_{t}^{\prime}$ for all $t \in \mathbb{N} \backslash\{1\}$ and (ii) $x_{1}=y_{1}$ and $x_{1}^{\prime}=y_{1}^{\prime}$, $\mathbf{x} \succsim \mathbf{y}$ if and only if $\mathbf{x}^{\prime} \succsim \mathbf{y}^{\prime}$.

Axiom RSEF is Postulate 3b in Koopmans' (1960) characterization of discounted utilitarianism restricted to the set of non-decreasing streams. It follows from axiom RSE by setting $\mathcal{T}=\{2,3, \ldots\}$.

Axiom RST (Restricted Stationarity) For any $\mathbf{x}, \mathbf{y}, \in \mathbf{X}^{+}$, there exists $z \in \mathbb{R}_{+}$ with $z \leq \min \left(x_{1}, y_{1}\right)$ such that $(z, \mathbf{x}) \succsim(z, \mathbf{y})$ if and only if $\mathbf{x} \succsim \mathbf{y}$.

Axiom RST is Koopmans' (1960) stationarity postulate (Postulate 4) restricted to the set of non-decreasing streams. The conjunction of axioms RSEF and RST is the restriction of axiom IF (Independent Future), as used by Asheim, Mitra and Tungodden (2010), to the set of non-decreasing streams.

Finally, we state the strong axiom of procedural equity, requiring social indifference with respect to all permutations $\pi \in \Pi$.

Axiom SA (Strong Anonymity) For any $\pi \in \Pi$ and $\mathbf{x} \in \mathbf{X}, \mathbf{x} \sim \mathbf{x}_{\pi}$. 


\subsection{Characterization}

In this subsection we characterize the class of SWOs satisfying $\mathbf{O}, \mathbf{C}, \mathbf{M}, \mathbf{R D}$, RSEP, RSEF, RST and SA. As a first step, we do so within the restricted domain $\overline{\mathbf{X}}$ of allocations that can be reordered into non-decreasing streams.

Definition 1 Rank-Discounted Utilitarian SWO. An SWR on $\overline{\mathbf{X}}$ is a RankDiscounted Utilitarian SWO (RDU SWO) if it is represented by an SWF $\bar{W}$ : $\overline{\mathrm{X}} \rightarrow \mathbb{R}$ defined by:

$$
\bar{W}(\mathbf{x})=(1-\beta) \sum_{r \in \mathbb{N}} \beta^{r-1} u\left(x_{[r]}\right),
$$

where $0<\beta<1$ is a real number and the function $u$ is continuous and increasing.

Although the RDU criterion can be seen as an infinite extension of families of single-series Ginis, as axiomatized by Bossert (1990), with the Gini weight of rank $r$ set equal to $\beta^{r-1}$, our axiomatization differs from Bossert's. The recursive methods that we use are similar to his recursivity property. However, we do not need the linear homogeneity and translatability properties which are essential for his result. We rely instead on RSEF and RST which are taken from intertemporal choice theory. ${ }^{2}$

Proposition 2. If an $S W R \succsim$ on $\overline{\mathrm{X}}$ satisfies axioms $\boldsymbol{O}, \boldsymbol{C}, \boldsymbol{M}, \boldsymbol{R D}, \boldsymbol{R S E P}$, $\boldsymbol{R} \boldsymbol{S E F}, \boldsymbol{R S} \boldsymbol{T}$ and $\boldsymbol{S A}$, then it is an $R D U S W O$.

Proof. See Appendix A for a simplified version of Koopmans' (1960) proof, similar to the one in Bleichrodt, Rhode and Wakker (2008). The proof is applied to non-decreasing streams, requiring the use of techniques developed by Wakker (1993) for additive representation of preferences on rank-ordered sets. Axiom C allows us to extend from a finite number of period to an infinite number of periods the representation on non-decreasing streams. Axiom SA allows us to extend the representation to the whole set $\overline{\mathbf{X}}$.

We then turn to the demonstration of the result that this class can be characterized in terms of extended RDU SWOs on the unrestricted domain $\mathbf{X}$.

Definition 2 Extended Rank-Discounted Utilitarian SWO. An SWR on $\mathbf{X}$ is an Extended Rank-Discounted Utilitarian SWO (ERDU SWO) if it is represented by an SWF $W: \mathbf{X} \rightarrow \mathbb{R}$ defined by:

$$
W(\mathbf{x})=u(\ell(\mathbf{x}))+(1-\beta) \sum_{r=1}^{|L(\mathbf{x})|} \beta^{r-1}\left(u\left(x_{[r]}\right)-u(\ell(\mathbf{x}))\right),
$$

${ }^{2}$ Another class of single-series Ginis is the class of single-parameter Ginis axiomatized by Donaldson and Weymark (1980), whose generalization in a continuous framework is presented in Donaldson and Weymark (1983). The finite population counterparts of the RDU criterion does not satisfy the principle of population which characterizes single-parameter Ginis. 
where $0<\beta<1$ is a real number and the function $u$ is continuous and increasing.

To investigate how the ERDU SWF $W$ extends the RDU SWF $\bar{W}$, define, for any $\mathbf{x} \in \mathbf{X}, \overline{\mathbf{x}}$ as follows:

$\left\{\begin{array}{l}\bar{x}_{t}=\min \left\{x_{t}, \ell(\mathbf{x})\right\} \text { for all } t \in \mathbb{N} \text { if }|L(\mathbf{x})|<+\infty \\ \overline{\mathbf{x}} \text { is the subsequence of } \mathbf{x} \text { consisting of all } x_{t} \text { with } t \in L(\mathbf{x}) \text { if }|L(\mathbf{x})|=+\infty\end{array}\right.$

Proposition 1 implies that, by construction, $\overline{\mathbf{x}}$ belongs to $\overline{\mathbf{X}}$; therefore, $\overline{\mathbf{x}}_{[]}$is well-defined. It follows from (2) and (3) that for all $\mathbf{x} \in \mathbf{X}$,

$$
W(\mathbf{x})=\bar{W}(\overline{\mathbf{x}})
$$

The ERDU SWF $W$ is consistent with the idea of constant rank-dependent discounting: any generation $t$ with $x_{t}>\ell(\mathbf{x})$ if $|L(\mathbf{x})|<+\infty$ or $x_{t} \geq \ell(\mathbf{x})$ if $|L(\mathbf{x})|=+\infty$ is infinitely ranked when consumption levels are ordered in a non-decreasing sequence, in the sense that there are infinitely many generations $t^{\prime}$ with $x_{t^{\prime}}<x_{t}$. Hence, no weight is placed on their marginal consumption.

Lemma 2. Assume that an $S W R \succsim$ satisfies $\boldsymbol{O}, \boldsymbol{C}, \boldsymbol{M}$ and $\boldsymbol{R} \boldsymbol{D}$, and is represented on $\overline{\mathbf{X}}$ by an $R D U S W F$. Then the $S W R \succsim$ is represented on $\mathbf{X}$ by an SWF $\widetilde{W}$ which coincides with $\bar{W}$ on $\overline{\mathbf{X}}$.

Proof. By $\mathbf{O}, \mathbf{C}, \mathbf{M}$ and $\mathbf{R D}$, for all $\mathbf{x} \in \mathbf{X}$ there exists a unique scalar $x_{e}$ such that $\mathbf{x}_{\mathbf{e}}^{\mathbf{c}} \sim \mathbf{x}$. The scalar $x_{e}$ is an equally distributed equivalent and it is a representation of $\succsim$. The SWO $\succsim$ is also represented on $\overline{\mathbf{X}}$ by an RDU $\operatorname{SWF}(1-\beta) \sum_{r \in \mathbb{N}} \beta^{r-1} u\left(x_{[r]}\right)$ where $0<\beta<1$ and $u$ is increasing. Define $\widetilde{W}(\mathbf{x})=u\left(x_{e}\right)$ for all $\mathbf{x} \in \mathbf{X}$. Since $u$ is increasing, $\widetilde{W}$ is also a representation of $\succsim$ and, by definition of the equally distributed equivalent on $\overline{\mathbf{X}}$, it is such that $\widetilde{W}(\mathbf{x})=(1-\beta) \sum_{r \in \mathbb{N}} \beta^{r-1} u\left(x_{[r]}\right)$ for all $\mathbf{x} \in \overline{\mathbf{X}}$.

Lemma 3. Assume that an $S W R \succsim$ satisfies $\boldsymbol{M}$ and $\boldsymbol{S} \boldsymbol{A}$, and is represented on $\overline{\mathbf{X}}$ by $\bar{W}$ and on $\mathbf{X}$ by $\widetilde{W}$. Then, for all $\mathbf{x} \in \mathbf{X}$ with $|L(\mathbf{x})| \geq T \geq 0$,

$$
\widetilde{W}(\mathbf{x}) \leq \bar{W}\left(x_{\pi(1)}, x_{\pi(2)}, \ldots, x_{\pi(T)}, \ell(\mathbf{x}), \ell(\mathbf{x}), \ldots\right)
$$

where, $\forall t \in\{1, \ldots, T\}, x_{\pi(t)}<\ell(\mathbf{x})$.

Proof. Such insensitivity for $x_{t}>\ell(\mathbf{x})$ is shown in Appendix A.

Lemma 4. Assume that an $S W R \succsim$ satisfies $\boldsymbol{M}$ and $\boldsymbol{S A}$, and is represented on $\overline{\mathbf{X}}$ by $\bar{W}$ and on $\mathbf{X}$ by $\widetilde{W}$. Then, for all $\mathbf{x} \in \mathbf{X}, \widetilde{W}(\mathbf{x})=\bar{W}(\overline{\mathbf{x}})=W(\mathbf{x})$.

Proof. This follows from Lemma 3; see Appendix A. 
Proposition 3. Assume that an $S W R \succsim$ satisfies $\boldsymbol{O}, \boldsymbol{C}, \boldsymbol{M}, \boldsymbol{R \boldsymbol { D }}$ and $\boldsymbol{S A}$, and is represented on $\overline{\mathbf{X}}$ by an $R D U S W F$. Then the $S W R \succsim$ is represented on $\mathbf{X}$ by an ERDU SWF.

Proof. This result follows from Lemmata 2 and 4.

Theorem 1. Consider an $S W R \succsim$ on $\mathbf{X}$. The following two statements are equivalent.

(1) $\succsim$ satisfies axioms $\boldsymbol{O}, \boldsymbol{C}, \boldsymbol{M}, \boldsymbol{R D}, \boldsymbol{R S E P}, \boldsymbol{R S E F}, \boldsymbol{R S T}$ and $\boldsymbol{S A}$.

(2) $\succsim$ is an ERDU SWO.

Proof. (1) implies (2). This follows from Propositions 2 and 3. (2) implies (1). This is easy to establish, and its proof is left to the reader.

\subsection{Properties}

By combining axioms $\mathbf{O}, \mathbf{C}, \mathbf{M}$ and $\mathbf{R D}$ with the unrestricted versions of separability of the present and future and stationarity - SEP, SEF and ST one obtains a characterization of discounted utilitarianism (DU), whereby all streams $\mathbf{X}$ in $\mathbf{X}$ are ranked according to the SWF (1) (cf. Asheim, Mitra and Tungodden, 2010, Proposition 9). DU does not satisfy SA as an axiom of procedural equity, since the permutation of consumption may change the DU social welfare. Moreover, as pointed out by Asheim, Mitra and Tungodden (2010), the DU SWF does not satisfy the following distributional equity axiom, giving priority to the future in conflicts where the present is better off than the future.

Axiom HEF (Hammond Equity for the Future) For all $x, y, w, z \in \mathbb{R}_{+}$, if $x>y>w>z$, then $\left(y, \mathbf{w}^{\mathbf{c}}\right) \succsim\left(x, \mathbf{z}^{\mathbf{c}}\right)$.

Finally, as pointed out by Chichilnisky (1996), DU is a dictatorship of the present, which on the domain $\mathbf{X}$ can be formalized as follows:

Axiom DP (Dictatorship of the Present) For all $\mathbf{x}, \mathbf{y} \in \mathbf{X}$ such that $\mathbf{x} \succ \mathbf{y}$, there exist $z \in \mathbb{R}_{+}$with $x_{t}, y_{t} \leq z$ for all $t \in \mathbb{N}$ and $T^{\prime} \in \mathbb{N}$ such that, for any $\mathbf{x}^{\prime}, \mathbf{y}^{\prime} \in[0, z]^{\mathbb{N}},\left(\mathbf{x}_{\mathbf{T}},,_{\mathbf{T}+1} \mathbf{x}^{\prime}\right) \succ\left(\mathbf{y}_{\mathbf{T}}, \mathbf{T}+\mathbf{1} \mathbf{y}^{\prime}\right)$ for all $T \geq T^{\prime}$.

Hence, a setting where $\mathbf{O}, \mathbf{C}, \mathbf{M}$ and $\mathbf{R D}$ are invoked, at least one of the axioms SEP, SEF or ST must be weakened to prevent such a dictatorship.

Axiom NDP (Non Dictatorship of the Present) Condition DP does not hold.

Chichilnisky (1996) allows for axiom NDP by dropping axiom ST in the class of sustainable preferences characterized by her Theorem 2. However, SWOs in this class do not satisfy the two other ethical axioms: HEF and SA. 
Building on Asheim, Mitra and Tungodden's (2010) axiomatic analysis of sustainable recursive SWFs, Asheim and Mitra (2010) allow for HEF by weakening SEP to RSEP (i.e., restricting separable present to the set of non-decreasing streams) in their analysis of sustainable discounted utilitarian (SDU) SWOs, while retaining the remaining axioms of the above axiomatization of DU. Moreover, SDU SWOs satisfy axiom NDP, but fail to satisfy axiom SA.

On this background it is of interest to note the following proposition.

Proposition 4. An ERDU SWO satisfies $\boldsymbol{H E F}$ and $\boldsymbol{N D P}$.

Proof. An ERDU SWO satisfies $\boldsymbol{H E F}$. Let $x>y>w>z \geq 0$. Then $W\left(y, \mathbf{w}^{\mathbf{c}}\right)$ $=u(w)>u(z)=W\left(x, \mathbf{z}^{\mathbf{c}}\right)$. An ERDU SWO satisfies $\boldsymbol{N D P}$. Let $\mathbf{x} \succ \mathbf{y}$. Choose any $z \geq 0$ satisfying $x_{t}, y_{t} \leq z$ for all $t \in \mathbb{N}$. Let $\mathbf{x}^{\prime}=\mathbf{y}^{\prime}=\mathbf{0}^{\mathbf{c}} \in[0, z]^{\mathbb{N}}$. Then $W\left(\mathbf{x}_{\mathbf{T}}, \mathbf{T}+\mathbf{1} \mathbf{x}^{\prime}\right)=W\left(\mathbf{y}_{\mathbf{T}}, \mathbf{T}+\mathbf{1} \mathbf{y}^{\prime}\right)$ for all $T \geq 0$.

Hence, when moving from SDU to ERDU, SA is added and SEF and ST are weakened to RSEF and RST. The weakening of ST to RST means that we lose time-consistency when social preferences are time-invariant. Even though time-inconsistency turns out not to be an issue when ERDU SWFs are applied to the Ramsey and Dasgupta-Heal-Solow growth models, as we do in Section 6 , it might be a problem in other environments. It also excludes the use of recursive methods, e.g., when faced with uncertainty.

Still, it is remarkable that anonymity (even in its strongest form, SA, allowing infinite permutations) can be combined with numerical representability and some sensitivity to the interests of the present generation, as such attempts have not earlier lead to SWOs with attractive properties. Axiom SA is a basic form of procedural equity, corresponding to equal treatment of generations. In this sense it seems more fundamental than the distributional axiom HEF.

As pointed out by Van Liedekerke and Lauwers (1997), axiom SA is in conflict with the strong Pareto principle. Moreover, Basu and Mitra (2003) showed that even finite anonymity (i.e., anonymity in its weaker form, involving only finite permutations) rules out the strong Pareto principle when combined with numerical representability. Finally, Zame (2007) and Lauwers (2010) demonstrated that SWOs satisfying both finite anonymity and strong Pareto cannot be explicitly described.

Strong anonymity, i.e., axiom $\mathbf{S A}$, is even in conflict with the weak Pareto principle whereby one stream is preferred to another stream if the former has higher consumption than the latter at all times. This is demonstrated by the following adaptation of Fleurbaey and Michel's (2003) proof of their Theorem 1 
to a setting where streams are bounded: consider

$$
\begin{aligned}
& \mathbf{x}=\left(\frac{1}{3}, \frac{2}{3}, \frac{1}{4}, \frac{3}{4}, \ldots, \frac{1}{k+2}, \frac{k+1}{k+2}, \ldots\right) \\
& \mathbf{y}=\left(\frac{1}{4}, \frac{1}{3}, \frac{1}{5}, \frac{2}{3}, \ldots, \frac{1}{k+3}, \frac{k}{k+1}, \ldots\right),
\end{aligned}
$$

where by axiom $\mathbf{S A} \mathbf{x}$ is indifferent to $\mathbf{y}$ even though $x_{t}>y_{t}$ for all $t \in \mathbb{N}$.

Because ERDU SWOs satisfy axiom SA, it follows that they must be in conflict with even the weak Pareto principle on the full domain $\mathbf{X}$, which indeed is what Lemma 3 entails. However, an important feature of ERDU SWOs is that they satisfy the strong Pareto principle on the restricted set $\overline{\mathbf{X}}$ of streams that can permuted into non-decreasing streams. This means that ERDU SWOs retain sensitivity to the interest of any one generation as long as there is only a finite number of other generations with lower consumption levels. Moreover, they fulfill the separability axiom on the set $\mathbf{X}^{+}$of non-decreasing streams. These are straightforward consequences of $(3)$, so that no proof is provided.

Proposition 5. An ERDU SWO satisfies $\boldsymbol{R S P}$ and $\boldsymbol{R S E}$.

\section{Inequality aversion}

Up to now, we have addressed the issue of procedural equity and its compatibility with the sensitivity to the interests of each generation.

In this section, we introduce concerns for distributional equity. We will show that inequality aversion can be properly measured and compared within the ERDU class of preferences. The next two sections will then show that inequality aversion has significant policy implications.

\subsection{The Pigou-Dalton transfer principle and inequality aversion}

Following the practice of expressing distributional equity ideals by means of transfer axioms, we consider a weak form of the Pigou-Dalton transfer principle:

Axiom PDT (Pigou-Dalton Transfer Principle) For any $\mathbf{x}, \mathbf{y} \in \mathbf{X}$, if there exist $\varepsilon \in \mathbb{R}_{++}$and $\tau, \tau^{\prime} \in \mathbb{N}$ such that $\varepsilon \leq y_{\tau}+\varepsilon=x_{\tau} \leq x_{\tau^{\prime}}=y_{\tau^{\prime}}-\varepsilon$ and $y_{t}=x_{t}$ for all $t \neq \tau, \tau^{\prime}$, then $\mathbf{x} \succsim \mathbf{y}$.

In this section, we study the restrictions imposed by PDT on ERDU criteria. These restrictions hold on the rank-discount factor $\beta$ and on the utility function $u$ in Eq. (3). Write $\succsim_{\beta, u}$ for the ERDU SWO characterized by $\beta$ and $u$.

Introduce the following index of non-concavity of the function $u$ :

$$
\mathcal{C}_{u}=\sup _{0<\varepsilon \leq x \leq x^{\prime}} \frac{u\left(x^{\prime}+\varepsilon\right)-u\left(x^{\prime}\right)}{u(x)-u(x-\varepsilon)}
$$


As shown by Chateauneuf, Cohen and Meilijson (2005), this index has two interesting properties: (1) $\mathcal{C}_{u} \geq 1$, with $\mathcal{C}_{u}=1$ corresponding to $u$ being concave; (2) and, when $u$ is differentiable, $\mathcal{C}_{u}=\sup _{y \leq x}\left(u^{\prime}(x) / u^{\prime}(y)\right)$.

The non-concavity index $\mathcal{C}_{u}$ and the discount factor $\beta$ jointly characterize ERDU SWFs satisfying the Pigou-Dalton transfer principle.

Proposition 6. An ERDU $S W O \succsim_{\beta, u}$ on $\mathbf{X}$ satisfies $\boldsymbol{P D T}$ if and only if

$$
\beta \times \mathcal{C}_{u} \leq 1
$$

Proof. See Appendix A.

Condition $\beta \times \mathcal{C}_{u} \leq 1$ means that the utility function $u$ must not be 'too nonconcave'. The concavity of $u$, though sufficient, is not necessary for an ERDU SWO to satisfy PDT.

In applications, it is convenient to consider the more specific class of homothetic ERDU SWOs which yield clear-cuts results for comparisons of inequality aversions and for the expression of the discount rate.

Definition 3 Homothetic Extended Rank-Discounted Utilitarian SWO. An SWO $\succsim$ on $\mathbf{X}$ is a homothetic extended rank-discounted utilitarian SWO (HERDU $\mathrm{SWO})$ if it can be represented by an SWF $W: \mathbf{X} \rightarrow \mathbb{R}$ defined by:

$$
W(\mathbf{x})= \begin{cases}\frac{(\ell(\mathbf{x}))^{1-\eta}}{1-\eta}+(1-\beta) \sum_{r \in \mathbb{N}} \beta^{r-1}\left(\frac{x_{[r]}^{1-\eta}}{1-\eta}-\frac{(\ell(\mathbf{x}))^{1-\eta}}{1-\eta}\right) & \text { if } \eta \neq 1 \\ \ln \ell(\mathbf{x})+(1-\beta) \sum_{r \in \mathbb{N}} \beta^{r-1}\left(\ln x_{[r]}-\ln \ell(\mathbf{x})\right) & \text { if } \eta=1\end{cases}
$$

where $0<\beta<1$ is a real number.

Denote by $\succsim_{\beta, \eta}$ a HERDU SWO represented by an SWR $W$ with rankdiscount factor $\beta$ and utility function $u(x)=x^{1-\eta} /(1-\eta)($ or $u(x)=\ln x$ if $\eta=1$ ). In contrast to the general case, the (weak) concavity of $u$ is necessary and sufficient for a HERDU SWOs to be inequality averse. For a HERDU SWO it is indeed the case that $\mathcal{C}_{u}=1$ whenever $\eta \geq 0$ and $\mathcal{C}_{u}=+\infty$ whenever $\eta<0$. This is summarized in the following corollary:

Corollary 1. A HERDU SWO on $\mathbf{X} \succsim_{\beta, \eta}$ satisfies $\boldsymbol{P D T}$ if and only if $\eta \geq 0$.

\subsection{Comparative inequality aversion}

Ranking different criteria according to the strength of their concerns for equality is an important prerequisite to study the policy implications of inequality aversion. The common way to do so is to define and compare the degree of inequality aversion of the underlying SWOs. The aim of this section is to perform such comparisons in the case of ERDU SWOs. 
We follow the procedure proposed in the literature on risk/uncertainty aversion to make such comparisons (see Grant and Quiggin, 2005). It consists in: (i) defining an inequality relation $\succ_{I}$; (ii) declaring an SWO $\succsim$ at least as inequality averse as an SWO $\widehat{\succsim}$ if, for any allocation $\mathbf{y}$, whenever a less unequal allocation $\mathbf{x}$ (according to $\succ_{I}$ ) is preferred to $\mathbf{y}$ according to $\widehat{\succsim}$, then $\mathbf{x}$ is also preferred to $\mathbf{y}$ according to $\succsim$.

We use a simple definition of the relation 'more unequal than' based on the notion of a 'local increase' in inequality, namely an inequality change affecting only two generations and leaving generations' ranks unchanged.

Definition 4. For any $\mathbf{x}, \mathbf{y} \in \mathbf{X}$, allocation $\mathbf{y}$ represents an elementary increase in inequality with respect to allocation $\mathbf{x}$, denoted $\mathbf{y} \succ_{I} \mathbf{x}$, if there exist $\varepsilon$, $\varepsilon^{\prime} \in \mathbb{R}_{++}$and $\tau, \tau^{\prime} \in \mathbb{N}$ such that $y_{\tau}+\varepsilon=x_{\tau} \leq x_{\tau^{\prime}}=y_{\tau^{\prime}}-\varepsilon^{\prime}, r_{\tau}(\mathbf{y})=r_{\tau}(\mathbf{x})$, $\bar{r}_{\tau^{\prime}}(\mathbf{y})=\bar{r}_{\tau^{\prime}}(\mathbf{x})$, and $y_{t}=x_{t}$ for all $t \neq \tau, \tau^{\prime}$.

The inequality relation $\succ_{I}$ is used to define comparative inequality aversion:

Definition 5. An SWO $\succsim$ is at least as inequality averse as an SWO $\widehat{\succsim}$ if, for any $\mathbf{x}$ and any $\mathbf{y} \succ_{I} \mathbf{x}$ : (i) $\mathbf{x} \widehat{\succsim} \mathbf{y} \Longrightarrow \mathbf{x} \succsim \mathbf{y}$, and (ii) $\mathbf{x} \widehat{\succ} \mathbf{y} \Longrightarrow \mathbf{x} \succ \mathbf{y}$.

Consider two ERDU SWOs, $\succsim_{\beta, u}$ and $\succsim_{\hat{\beta}, \hat{u}}$. To assess their relative inequality aversion, the discount factors $\beta$ and $\hat{\beta}$ and the relative concavity of the utility functions $u$ and $\hat{u}$ must be compared. The following two indices do so:

$$
\begin{aligned}
& \mathcal{D}_{\beta, \hat{\beta}}=\inf _{t<t^{\prime}} \frac{\beta^{t} / \hat{\beta}^{t}}{\beta^{t^{\prime}} / \hat{\beta}^{t^{\prime}}}= \begin{cases}\hat{\beta} / \beta & \text { if } \beta \leq \hat{\beta} \\
0 & \text { if } \beta>\hat{\beta}\end{cases} \\
& \mathcal{C}_{u, \hat{u}}=\sup _{0 \leq x_{1}<x_{2} \leq x_{3}<x_{4}} \frac{\left[u\left(x_{4}\right)-u\left(x_{3}\right)\right] /\left[\hat{u}\left(x_{4}\right)-\hat{u}\left(x_{3}\right)\right]}{\left[u\left(x_{2}\right)-u\left(x_{1}\right)\right] /\left[\hat{u}\left(x_{2}\right)-\hat{u}\left(x_{1}\right)\right]} .
\end{aligned}
$$

The index $\mathcal{D}_{\beta, \hat{\beta}}$ is an index of the relative decreasing speed of the social weights. The faster the social weights decrease, the less the society cares for better off generations. The index $\mathcal{C}_{u, \hat{u}}$ is an index of relative concavity of the utility functions $u$ and $\hat{u}$. Furthermore (Grant and Quiggin, 2005), $\mathcal{C}_{u, \hat{u}} \geq 1$, with $\mathcal{C}_{u, \hat{u}}=1$ corresponding to the case where $u$ is an increasing concave transformation of $\hat{u}$. In addition, if $u$ and $\hat{u}$ are differentiable, $\mathcal{C}_{u, \hat{u}}=\sup _{y \leq x}\left(u^{\prime}(x) \hat{u}^{\prime}(y)\right) /\left(u^{\prime}(y) \hat{u}^{\prime}(x)\right)$.

The comparative inequality aversion of two ERDU SWOs can be characterized as follows:

Proposition 7. Consider two ERDU SWOs, $\succsim_{\beta, u}$ and $\succsim_{\hat{\beta}, \hat{u}}$, on $\mathbf{X}$. Then $\succsim_{\beta, u}$ is at least as inequality averse as $\succsim_{\hat{\beta}, \hat{u}}$ if and only if

$$
\mathcal{D}_{\beta, \hat{\beta}} \geq \mathcal{C}_{u, \hat{u}}
$$


Proof. See Appendix A.

By Proposition $7, \beta \leq \hat{\beta}$ is a necessary condition for $\succsim_{\beta, u}$ to be at least as inequality averse as $\succsim_{\hat{\beta}, \hat{u}}$. A more inequality averse ERDU social observer has a lower rank-discount factor and thus discount more the utility of better off generations. Moreover, if $\beta=\hat{\beta}$, then $u$ must be a concave transformation of $\hat{u}$.

Even clearer results can be obtained in the case of HERDU SWOs. Indeed, it is straightforward that, whenever $u(x)=x^{1-\eta} /(1-\eta)$ and $\hat{u}(x)=x^{1-\hat{\eta}} /(1-\hat{\eta})$, $\mathcal{C}_{u, \hat{u}}=1$ if $\eta \geq \hat{\eta}$, and $\mathcal{C}_{u, \hat{u}}=+\infty$ if $\eta<\hat{\eta}$. We hence obtain the following simple conditions for comparative inequality aversion of HERDU SWOs:

Corollary 2. Consider two HERDU SWOs, $\succsim_{\beta, \eta}$ and $\succsim_{\hat{\beta}, \hat{\eta}}$, on $\mathbf{X}$. Then $\succsim_{\beta, \eta}$ is at least as inequality averse as $\succsim_{\hat{\beta}, \hat{\eta}}$ if and only if $\beta \leq \hat{\beta}$ and $\eta \geq \hat{\eta}$.

As in the static case, inequality aversion is a key policy parameter in intertemporal problems, playing an important role in designing optimal policies. In Section 5, we describe how it affects social discounting, while in Section 6, we study optimal ERDU policies and highlight the impact of inequality aversion.

\section{Rank-discounted utilitarianism and social discounting}

Triggered by the Stern (2006) review of climate change, the social discount rate has attracted much attention in recent years (Nordhaus, 2007; Weitzman, 2007; Dasgupta, 2008). The controversy has not held on the social welfare function used to assess different streams, as all the authors have endorsed the DU approach. The controversy has held on the value of the parameters in the DU SWF (1). In particular, the time-discount rate $\beta$ and the elasticity of marginal utility, $\eta_{u}(x)=-d \ln u^{\prime}(x) / d \ln x$, have a critical role in the determination of the social discount rate. However, there has been no consensus on the interpretation and the value of these key parameters.

In this section, we derive the social discount rate arising from ERDU SWOs. Doing so we prove that the key parameters of the social discount rate have interpretations in terms of inequality aversion.

Assume that an ERDU SWO $\succsim_{\beta, u}$, has the property the function $u$ in Eq. (3) is twice continuously differentiable. In that case $\succsim_{\beta, u}$ is said to be a smooth ERDU SWO. Also, consider consumption streams $\mathbf{x}$ in $\mathbf{X}$ where

(i) $|L(\mathbf{x})|<+\infty$ and $x_{t} \neq \ell(\mathbf{x})$ for all $t \in \mathbb{N}$, or $|L(\mathbf{x})|=+\infty$,

(ii) no pair in $L(\mathbf{x})$ has the same consumption level (i.e., $x_{t} \neq x_{\tau}$ if $t, \tau \in L(\mathbf{x})$ ).

The set of such streams is denoted $\mathbf{X}_{\neq}$. Any stream in $\mathbf{X}_{\neq}$has the property that $r_{t}(\mathbf{x})=\bar{r}_{t}(\mathbf{x})<+\infty$ if $t \in L(\mathbf{x})$, while $r_{t}(\mathbf{x})=\bar{r}_{t}(\mathbf{x})=+\infty$ otherwise. An 
SWR $W$ representing a smooth ERDU SWO is differentiable on $\mathbf{X}_{\neq}$only, with $\partial W(\mathbf{x}) / \partial x_{t}=\beta^{r_{t}(\mathbf{x})-1} u^{\prime}\left(x_{t}\right)>0$ if $t \in L(\mathbf{x})$ and $\partial W(\mathbf{x}) / \partial x_{t}=0$ otherwise.

The social discount rate evaluates how much an increase in marginal consumption in period $t$ is 'worth' in terms of first period consumption. It is given by the following formal expression: ${ }^{3}$

Definition 6. Let $W$ be the SWF used to evaluate policies. Then the social discount rate at period $t$ for a stream $\mathbf{x}$ is:

$$
\rho_{t}(\mathbf{x})=\frac{\ln \left(\partial W / \partial x_{1}\right)-\ln \left(\partial W / \partial x_{t}\right)}{t-1} .
$$

Consider a smooth ERDU SWO $\succsim_{\beta, u}$ and denote by $\delta=-\ln \beta$ the rank discount rate. Also denote by $g_{t}(\mathbf{x})$ the average per period growth rate between 1 and $t: g_{t}(\mathbf{x})=\left(\ln x_{t}-\ln x_{1}\right) /(t-1)$. The social discount rate arising from a smooth ERDU SWO can now be approximated:

Proposition 8. Let $\succsim_{\beta, u}$ be a smooth ERDU SWO, and consider a stream $\mathbf{x} \in$ $\mathbf{X}_{\neq}$with $1 \in L(\mathbf{x})$. Then the social discount rate, $\rho_{t}(\mathbf{x})$, at period $t \in L(\mathbf{x}) \backslash\{1\}$ is approximated by the rhs. of the following expression:

$$
\rho_{t}(\mathbf{x}) \approx \frac{r_{t}(\mathbf{x})-r_{1}(\mathbf{x})}{t-1} \delta+\eta_{u}\left(x_{1}\right) g_{t}(\mathbf{x})
$$

Proof. From the ERDU SWO it follows that

$$
\begin{aligned}
\rho_{t}(\mathbf{x}) & =\frac{\left(\left(r_{1}(\mathbf{x})-1\right) \ln \beta+\ln \left(u^{\prime}\left(x_{1}\right)\right)\right)-\left(\left(r_{t}(\mathbf{x})-1\right) \ln \beta+\ln \left(u^{\prime}\left(x_{t}\right)\right)\right)}{t-1} \\
& =\frac{r_{t}(\mathbf{x})-r_{1}(\mathbf{x})}{t-1} \delta+\frac{\ln u^{\prime}\left(x_{1}\right)-\ln u^{\prime}\left(x_{t}\right)}{t-1} \\
& \approx \frac{r_{t}(\mathbf{x})-r_{1}(\mathbf{x})}{t-1} \delta-\frac{d \ln u^{\prime}\left(x_{1}\right)}{d \ln x_{1}} \cdot \frac{\ln x_{t}-\ln x_{1}}{t-1} \\
& =\frac{r_{t}(\mathbf{x})-r_{1}(\mathbf{x})}{t-1} \delta+\eta_{u}\left(x_{1}\right) g_{t}(\mathbf{x}),
\end{aligned}
$$

using a log-linear approximation for $u^{\prime}(x)$.

\footnotetext{
${ }^{3}$ To understand the expression, imagine that today the society makes a marginal investment $\varepsilon$ whose rate of return is $\rho$, so that the generation born in period $t$ can consume $e^{\rho(t-1)} \varepsilon$ more units of aggregate good. The change in social welfare through this investment is:

$$
d W(\mathbf{x})=\frac{\partial W}{\partial x_{t}} e^{\rho(t-1)} \varepsilon-\frac{\partial W}{\partial x_{1}} \varepsilon
$$

The social discount rate is the rate of return that makes the change in social welfare nil.
} 
Approximation (6) shows that the social discount rate is rank-dependent: it depends crucially on the distance between the welfare rank of generation $t$ and the one of the first generation. The further generation $t$ is in the intergenerational distribution, the larger the social discount rate, and vice versa.

This remark leads to a second insight. If generation $t$ is worse off than the first generation, the social discount rate will be negative, provided that $\eta_{u}\left(x_{1}\right) \geq 0$, which is always the case when $u$ is concave. It has been pointed out in the literature using a DU approach that the social discount rate may be negative when future generations are sufficiently worse off (see for instance Dasgupta, 2008, p. 150). With ERDU, this should always be the case as soon as a future generations are worse off and the function $u$ is concave.

On the set of increasing consumption streams, the familiar expression $\rho_{t}(\mathbf{x}) \approx$ $\delta+\eta_{u}\left(x_{1}\right) g_{t}(\mathbf{x})$ is obtained. For smooth HERDU SWOs, the log-linear approximation of marginal utility is exact and the expression becomes $\rho_{t}(\mathbf{x})=\delta+\eta g_{t}(\mathbf{x})$. This expression emphasizes the crucial role played by the ethical parameters to determine the social discount rate. Indeed, $\delta$ and $\eta$ conjointly characterize the attitude towards inequality: a more inequality averse social observer should have a higher $\delta$ (lower $\beta$ ) and/or a higher $\eta$. Therefore, a more inequality averse society should discount the future more whenever future generations are better off. This insight actually generalizes to all ERDU SWOs.

Proposition 9. Consider two smooth ERDU $S W O s, \succsim_{\beta, u}$ and $\succsim_{\hat{\beta}, \hat{u}}$, and a stream $\mathbf{x} \in \mathbf{X}_{\neq}$with $1 \in L(\mathbf{x})$. Let $\rho_{t}(\mathbf{x})$ and $\hat{\rho}_{t}(\mathbf{x})$ be the associated discount rates at period $t \in L(\mathbf{x}) \backslash\{1\}$. If $\succsim_{\beta, u}$ is at least as inequality averse as $\succsim_{\hat{\beta}, \hat{u}}$, then:

(1) $\rho_{t}(\mathbf{x}) \geq \hat{\rho}_{t}(\mathbf{x})$ if $x_{t}>x_{1}$.

(2) $\rho_{t}(\mathbf{x}) \leq \hat{\rho}_{t}(\mathbf{x})$ if $x_{t}<x_{1}$.

Proof. For any $\mathbf{x} \in \mathbf{X}_{\neq}$and any $t \in L(\mathbf{x}) \backslash\{1\}, \rho_{t}(\mathbf{x}) \geq \hat{\rho}_{t}(\mathbf{x})$ if and only if

$$
\Delta=\frac{\partial W / \partial x_{1}}{\partial W / \partial x_{t}}-\frac{\partial \widehat{W} / \partial x_{1}}{\partial \widehat{W} / \partial x_{t}}=\frac{\beta^{r_{1}(\mathbf{x})-1} u^{\prime}\left(x_{1}\right)}{\beta^{r_{t}(\mathbf{x})-1} u^{\prime}\left(x_{t}\right)}-\frac{\hat{\beta}^{r_{1}(\mathbf{x})-1} \hat{u}^{\prime}\left(x_{1}\right)}{\hat{\beta}^{r_{t}(\mathbf{x})-1} \hat{u}^{\prime}\left(x_{t}\right)} \geq 0,
$$

where $W(\widehat{W})$ represents $\succsim_{\beta, u}\left(\succsim_{\hat{\beta}, \hat{u}}\right)$. There are two ways to rearrange $\Delta$ :

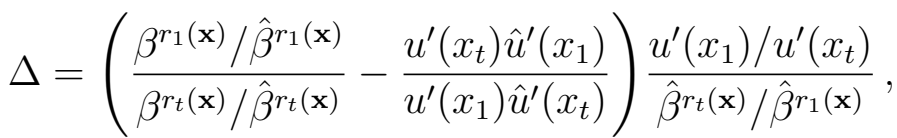

$$
\begin{aligned}
& \Delta=\left(\frac{u^{\prime}\left(x_{1}\right) \hat{u}^{\prime}\left(x_{t}\right)}{u^{\prime}\left(x_{t}\right) \hat{u}^{\prime}\left(x_{1}\right)}-\frac{\beta^{r_{t}(\mathbf{x})} / \hat{\beta}^{r_{t}(\mathbf{x})}}{\beta^{r_{1}(\mathbf{x}) / \hat{\beta}^{r_{1}(\mathbf{x})}}}\right) \frac{\hat{u}^{\prime}\left(x_{1}\right) / \hat{u}^{\prime}\left(x_{t}\right)}{\beta^{r_{t}(\mathbf{x})} / \beta^{r_{1}(\mathbf{x})}} .
\end{aligned}
$$


Using the definitions of $\mathcal{D}_{\beta, \hat{\beta}}$ and $\mathcal{C}_{u, \hat{u}}$, we obtain: by Eq. (7), for $x_{t}>x_{1}$,

$$
\Delta \geq\left(\mathcal{D}_{\beta, \hat{\beta}}-\mathcal{C}_{u, \hat{u}}\right)\left(u^{\prime}\left(x_{1}\right) / u^{\prime}\left(x_{t}\right)\right) /\left(\hat{\beta}^{r_{t}(\mathbf{x})} / \hat{\beta}^{r_{1}(\mathbf{x})}\right) \geq 0
$$

by Proposition 7, noting that $r_{t}(\mathbf{x})>r_{1}(\mathbf{x})$; by Eq. (8), for $x_{t}<x_{1}$,

$$
\Delta \leq\left(\mathcal{C}_{u, \hat{u}}-\mathcal{D}_{\beta, \hat{\beta}}\right)\left(\hat{u}^{\prime}\left(x_{1}\right) / \hat{u}^{\prime}\left(x_{t}\right)\right)\left(\beta^{r_{t}(\mathbf{x})} / \beta^{r_{1}(\mathbf{x})}\right) \leq 0
$$

by Proposition 7 , noting that $r_{t}(\mathbf{x})<r_{1}(\mathbf{x})$.

It is a strength of the class of HERDU criteria that the two parameters $\delta$ and $\eta$ have a consistent, common interpretation in terms of intergenerational inequality aversion. By increasing each of $\delta$ and $\eta$, inequality aversion is enhanced. For increasing streams, a more inequality averse society discounts the future more, with the discount rate having a clear ethical significance.

This is in contrast with the class of homothetic DU criteria where the two parameters $\delta$ and $\eta$ represent different ethical notions. The time discounting parameter $\delta$ measures the intensity of intergenerational (procedural) inequity. A fairer society should choose a lower $\delta$. On the other hand, the elasticity of marginal utility $\eta$ is often interpreted as a measure of intra-temporal inequality aversion. A more egalitarian society should choose a higher $\eta$. As a consequence, it is not clear what the social discount rate of an 'equity-minded' society should be: it should discount the future less to avoid intergenerational inequity, but discount the future more because it is more averse to intra-period inequalities.

The result in Proposition 9 has important policy implications, in particular for the question of climate change. If one believes that future generations will be better off in spite of climate change, ${ }^{4}$ then a more inequality averse ERDU social observer will agree with the recommendation of Nordhaus (2008) to have a gradual emissions-control policy with increasing carbon price rather than with that of Stern (2006) who calls for strong immediate action to mitigate climate change. Indeed, Nordhaus proposes to use $\delta=0.015$ and $\eta=2$ whereas Stern argues in favor of $\delta=0.001$ and $\eta=1$. However, the policy recommendation will be totally different if one believes that climate change might strongly affect the economy so that declining consumption would occur for some generations in the future. This perspective may not be unrealistic for some poor developing countries particularly exposed to climate change. In that case, an ERDU social observer using $\eta=1$ and $\delta>0$ will recommend discounting future consumption at a negative rate. This rate is lower than the one promoted by Stern for decreasing consumption streams, thus leading to even stronger action.

\footnotetext{
${ }^{4}$ The assumption is verified in the central scenario of most climate-economy integrated assessment models, such as the RICE model of Nordhaus (2008) and the PAGE model used in the Stern (2006) review.
} 


\section{Optimal rank-discounted utilitarian policies}

In this section, we establish that ERDU SWOs can be applied to two benchmark models — the Ramsey and Dasgupta-Heal-Solow (DHS) growth models — and show that the ERDU optimal streams in these models are the same as the ones promoted by the SDU SWOs recently studied by Asheim and Mitra (2010).

In these model, the ERDU optimal streams maximize DU welfare over all non-decreasing streams. By the justification of sustainability proposed by Asheim, Buchholz and Tungodden (2001), finite anonymity combined with the strong Pareto principle rules out all streams that are not non-decreasing when applied to 'productive' technologies. Morever, ERDU welfare coincides with DU welfare on the set of non-decreasing streams, thereby providing intuition for choosing streams that maximize DU welfare over all non-decreasing streams. However, Asheim, Buchholz and Tungodden's (2001) argument is not directly applicable here since (i) ERDU SWOs do not satisfy the strong Pareto principle for streams that cannot be reordered into non-decreasing streams, and (ii) the DHS growth model is 'productive' only if resource extraction is positive.

For this section, assume that the SWR $\succsim$ on the set of bounded consumption streams is an ERDU SWO represented by $W$, as defined by Definition 2 , where $u$ is assumed to strictly concave and continuously differentiable (on $\mathbb{R}_{++}$) with $\lim _{x \rightarrow 0} u^{\prime}(0)=+\infty$. These additional properties on $u$ do not follow from the axiomatic basis for ERDU SWOs, but is imposed on the SWO for the purpose of the analysis of this section. Write $\succsim_{\beta, u}$ for the ERDU SWO determined by $\beta$ and $u$, where the properties of $u$ are as described in this paragraph.

Both the Ramsey and DHS models allow for streams that are not bounded above, a complication that must be addressed. For an unbounded stream $\mathbf{x}$, $\ell(\mathbf{x})$ need not exist. If $\ell(\mathbf{x})$ does not exist, then $\mathbf{x}$ can be permuted into a nondecreasing stream, implying that Proposition 1 can be reformulated as follows on any set $\mathbf{X} \subseteq \mathbb{R}_{+}^{\mathbb{N}}$ where $\mathbf{X}$ admits elements that are not bounded above.

\section{Proposition $\mathbf{1}^{\prime}$.}

(a) If $\ell(\mathbf{x})$ does not exist for an allocation $\mathbf{x} \in \mathbf{X}$, then $\mathbf{x}$ belongs to $\overline{\mathbf{X}}$.

(b) If $\ell(\mathbf{x})$ exists for an allocation $\mathbf{x} \in \mathbf{X}$ and $|L(\mathbf{x})|<+\infty$, then $\mathbf{x}$ belongs to $\overline{\mathbf{X}}$ if and only if $x_{t} \leq \ell(\mathbf{x})$ for all $t \in \mathbb{N}$.

(c) If $\ell(\mathbf{x})$ exists for an allocation $\mathbf{x} \in \mathbf{X}$ and $|L(\mathbf{x})|=+\infty$, then $\mathbf{x}$ belongs to $\overline{\mathbf{X}}$ if and only if $x_{t}<\ell(\mathbf{x})$ for all $t \in \mathbb{N}$.

Let $W$ be defined by $W(\mathbf{x})=\bar{W}(\mathbf{x})$ (cf. Definition 1 ) if $\ell(\mathbf{x})$ does not exist, while $W$ is defined by Definition 2 if $\ell(\mathbf{x})$ exists. Then $W(\mathbf{x})=\bar{W}(\overline{\mathbf{x}})$ (cf. Eq. (4)) still holds where $\overline{\mathbf{x}}=\mathbf{x}$ whenever $\ell(\mathbf{x})$ does not exist.

As shown by Lemmata 1 and 2 in Asheim and Mitra (2010), in our applications the unilateral Laplace transform $\left(\sum_{t \in \mathbb{N}} \beta^{t-1} x_{t}\right)$ is finite for any $0<\beta<1$ 
and any feasible $\mathbf{x}$ in these technologies. We will see below that this implies that $W(\mathbf{x})$ is finite for all feasible streams. Hence, let $\mathbf{X}=\left\{\mathbf{x} \in \mathbb{R}_{+}^{\mathbb{N}} \mid W(\mathbf{x})<+\infty\right\}$ for this section, and let the SWR $\succsim$ on $\mathbf{X}$ be an ERDU SWO represented by $W$.

The two subsequent subsections introduce sets of feasible streams. A stream $\mathbf{x}$ is optimal if $\mathbf{x}$ is feasible and $W(\mathbf{x}) \geq W\left(\mathbf{x}^{\prime}\right)$ for all feasible streams $\mathbf{x}^{\prime}$.

\subsection{The Ramsey growth model}

Assume that the technology is given by a strictly increasing, concave, and continuously differentiable production function $f: \mathbb{R}_{+} \rightarrow \mathbb{R}_{+}$, satisfying $f(0)=0$ and $\lim _{k \rightarrow \infty} f^{\prime}(k)=0$. A consumption stream $\mathbf{x}=\left(x_{1}, x_{2}, \ldots\right)$ is feasible given an initial capital stock $k_{1}>0$ if there exists a stream $\left\{k_{2}, k_{3}, \ldots\right\}$ such that

$$
x_{t}+k_{t+1} \leq f\left(k_{t}\right)+k_{t}, \quad x_{t} \geq 0, \quad k_{t} \geq 0
$$

for all $t \in \mathbb{N}$. Such a technology is referred to as a Ramsey technology.

Lemma 5. If $\mathbf{x} \in \mathbf{X}$ is feasible in a Ramsey technology, then $\overline{\mathbf{x}}$ is also feasible. If $\mathbf{x} \in \overline{\mathbf{X}}$ is feasible in a Ramsey technology, then $\mathbf{x}_{[]}$is also feasible.

Proof. These results follow as storage is costless in a Ramsey technology; cf. Asheim (1991, Lemma 3).

It follows from Lemma 1 of Asheim and Mitra (2010) and Lemma 5 above, combined with the concavity of $u$, that $W(\mathbf{x})$ is finite for any feasible stream $\mathbf{x}$ in a Ramsey technology.

Following Asheim and Mitra (2010) (but changing notation slightly), define the gross output function as $g(k)=f(k)+k$, and denote by $x(y)$ the unique solution to the equation $y=g(y-x(y))$ such that $0 \leq x(y) \leq y$. The function $x(y)$ is well-defined, continuous and differentiable (see Asheim and Mitra, 2010). Write $y_{\infty}(\beta) \equiv \min \left\{y \geq 0 \mid \beta g^{\prime}(y-x(y)) \leq 1\right\}$. The function $y_{\infty}$ is strictly increasing for all $\beta$ satisfying that there exists $k \geq 0$ such that $\beta g^{\prime}(k)=1$ (Asheim and Mitra, 2010).

Proposition 10. Consider an ERDU $S W O \succsim_{\beta, u}$ where $u$ is assumed to be strictly concave and continuously differentiable (on $\mathbb{R}_{++}$) with $\lim _{x \rightarrow 0} u^{\prime}(0)=0$, a Ramsey technology, and an initial capital stock $k_{1}>1$. There exists a unique optimal consumption stream, denoted $\mathbf{x}^{*}$, which is characterized as follows:

(a) If $y_{1}=g\left(k_{1}\right) \geq y_{\infty}(\beta)$, then $\mathbf{x}^{*}$ is a stationary stream with $x_{t}^{*}=x\left(y_{1}\right)$ for all $t \geq 1$.

(b) If $y_{1}=g\left(k_{1}\right)<y_{\infty}(\beta)$, then $\mathbf{x}^{*}$ is an increasing stream, converging to $x\left(y_{\infty}(\beta)\right)$ and maximizing $(1-\beta) \sum_{t \in \mathbb{N}} \beta^{t-1} u\left(x_{t}\right)$ over all feasible streams. 
Proof. Step 1: If $\mathbf{x} \in \mathbf{X}$ is optimal, then $\overline{\mathbf{x}} \in \overline{\mathbf{X}}$ is also optimal. If $\mathbf{x}$ is optimal, then $\mathbf{x}$ is also feasible. By Lemma $5, \overline{\mathbf{x}}$ is also feasible. By $(4), W(\overline{\mathbf{x}})=\bar{W}(\overline{\mathbf{x}})=$ $W(\mathbf{x}) \geq W\left(\mathbf{x}^{\prime}\right)$ for all feasible streams $\mathbf{x}^{\prime}$. Hence, $\overline{\mathbf{x}} \in \overline{\mathbf{X}}$ is also optimal.

Step 2: If $\mathbf{x} \in \overline{\mathbf{X}}$ is optimal, then $\mathbf{x}_{[]} \in \mathbf{X}^{+}$is also optimal. If $\mathbf{x}$ is optimal, then $\mathbf{x}$ is also feasible. By Lemma $5, \mathbf{x}_{[]}$is also feasible. Since $\mathbf{x}_{[]}$is a permutation of $\mathbf{x}, W\left(\mathbf{x}_{[]}\right)=W(\mathbf{x}) \geq W\left(\mathbf{x}^{\prime}\right)$ for all feasible streams $\mathbf{x}^{\prime}$. Hence, $\mathbf{x}_{[]} \in \mathbf{X}^{+}$ is also optimal.

Step 3: If $\mathbf{x} \in \mathbf{X}^{+}$is optimal, then $\mathbf{x}$ is the efficient stream $\mathbf{x}^{*}$ characterized by (a) and (b). The optimality of a non-decreasing $\mathbf{x}$ implies that $\mathbf{x}$ maximizes $\bar{W}\left(\mathbf{x}^{\prime}\right)$ over all non-decreasing streams $\mathbf{x}^{\prime}$. By Proposition 6 of Asheim (1991), the efficient stream $\mathbf{x}^{*}$ characterized by (a) and (b) is the unique stream maximizing $\bar{W}\left(\mathbf{x}^{\prime}\right)$ over all non-decreasing streams $\mathbf{x}^{\prime}$.

Step 4: If $\mathbf{x} \in \overline{\mathbf{X}} \backslash \mathbf{X}^{+}$, then $\mathbf{x}$ is not optimal. Suppose $\mathbf{x} \in \overline{\mathbf{X}} \backslash \mathbf{X}^{+}$is optimal. By step 2, $\mathbf{x}_{[]} \in \mathbf{X}^{+}$is optimal. However, by step 3, if $\mathbf{x}_{[]} \in \mathbf{X}^{+}$is optimal, then $\mathbf{x}_{[]}$coincides with the efficient stream $\mathbf{x}^{*}$ characterized by (a) and (b) However, it is not feasible to permute the efficient $\mathbf{x}^{*} \in \mathbf{X}^{+}$into $\mathbf{x} \in \overline{\mathbf{X}} \backslash \mathbf{X}^{+}$, as this contradicts that acceleration of consumption along an efficient stream with positive capital stocks is costly in a Ramsey technology (Asheim, 1991, Lemma $3)$. Hence, $\mathbf{x}$ is not optimal.

Step 5: If $\mathbf{x} \in \mathbf{X} \backslash \overline{\mathbf{X}}$, then $\mathbf{x}$ is not optimal. Suppose $\mathbf{x} \in \mathbf{X} \backslash \overline{\mathbf{X}}$ is optimal. By step $1, \overline{\mathbf{x}} \in \overline{\mathbf{X}}$ is optimal and, by the property of costless augmentation of initial consumption (cf. Asheim, 1991, Lemma 3), inefficient. However, by steps 3 and 4 , if $\overline{\mathbf{x}} \in \overline{\mathbf{X}}$ is optimal, then $\overline{\mathbf{x}}$ coincides with the efficient stream $\mathbf{x}^{*}$ characterized by (a) and (b). This contradicts that $\mathbf{x}$ is optimal.

Step 6: The efficient stream $\mathbf{x}^{*}$ characterized by (a) and (b) is optimal. By Proposition 6 of Asheim (1991), $W\left(\mathbf{x}^{*}\right)=\bar{W}\left(\mathbf{x}^{*}\right) \geq \bar{W}(\mathbf{x})=W(\mathbf{x})$ if $\mathbf{x}$ is non-decreasing. If $\mathbf{x} \in \overline{\mathbf{X}} \backslash \mathbf{X}^{+}$, then by Lemma 5 the permutation of $\mathbf{x}$ into the non-decreasing stream $\mathbf{x}_{[]}$is feasible, and furthermore, $W\left(\mathbf{x}^{*}\right)=\bar{W}\left(\mathbf{x}^{*}\right) \geq$ $\bar{W}\left(\mathbf{x}_{[]}\right)=W\left(\mathbf{x}_{[]}\right)=W(\mathbf{x})$. Hence, $W\left(\mathbf{x}^{*}\right) \geq W(\mathbf{x})$ if $\mathbf{x} \in \overline{\mathbf{X}}$. If $\mathbf{x} \in \mathbf{X} \backslash \overline{\mathbf{X}}$, then $W\left(\mathbf{x}^{*}\right) \geq W(\overline{\mathbf{x}})=\bar{W}(\overline{\mathbf{x}})=W(\mathbf{x})$ by $(4)$, since $\overline{\mathbf{x}} \in \overline{\mathbf{X}}$.

Proposition 10 shows that ERDU preferences can be operationalized in the basic Ramsey model. We are able to characterize a unique optimal solution, which we call the sustainable discounted utilitarian solution, being is the same as in Asheim (1991) and Asheim and Mitra (2010).

Compared to SDU preferences, ERDU preferences emphasize more clearly the influence of inequality aversion on optimal policy. Indeed, we know that a necessary condition for an ERDU SWF $W_{\beta, u}$ to more inequality averse than another ERDU SWF $W_{\hat{\beta}, \hat{u}}$ is that $\beta \leq \hat{\beta}$. From Proposition 10, it follows that:

- A more inequality averse ERDU society $\succsim_{\beta, u}$ converges to a lower steady state consumption than a less inequality averse society $\succsim_{\hat{\beta}, \hat{u}}$ whenever $k_{1}$ 
satisfies $g\left(k_{1}\right)<y_{\infty}(\hat{\beta})$ and lead to the same steady state consumption otherwise.

- A more inequality averse ERDU society $\succsim_{\beta, u}$ prevents growth for a larger set of initial conditions than a less inequality averse society $\succsim_{\hat{\beta}, \hat{u}}$ (for $k_{1}$ satisfying $g\left(k_{1}\right) \geq y_{\infty}(\beta)$ as opposed to $k_{1}$ satisfying $\left.g\left(k_{1}\right) \geq y_{\infty}(\hat{\beta})\right)$.

Regarding the second point, recall that the maximin always prevents growth. The maximin is the special case of ERDU preferences where $\beta \rightarrow 0$, an extreme aversion to inequality. However, growth is also prevented for low values of $\beta$.

Inequality aversion therefore modifies both the long-run perspectives of the society and the prospects of an egalitarian (stationary) distribution. Only the parameter $\beta$ determines the long-term impact of inequality aversion. The other dimension of inequality aversion, the concavity of the function $u$, has only an impact on the speed of the convergence to the steady state when $g\left(k_{1}\right)<y_{\infty}(\beta)$.

\subsection{The Dasgupta-Heal-Solow growth model}

The Dasgupta-Heal-Solow model (Dasgupta and Heal, 1974; Solow, 1974) is the standard model of growth with an exhaustible natural resource. Production depends on a man-made physical capital $k_{t}^{m}$, on the extraction $d_{t}$ of a natural exhaustible resource $k_{t}^{n}$ and on the labor supply $\ell_{t}$. The natural resource is depleted by the resource use, so that $k_{t+1}^{n}=k_{t}^{n}-d_{t}$. The production function $\hat{f}\left(k_{t}^{m}, d_{t}, l_{t}\right)$ is concave, non-decreasing, homogeneous of degree one, and twice continuously differentiable. It satisfies $\left(\hat{f}_{k^{m}}, \hat{f}_{d}, \hat{f}_{\ell}\right) \gg 0$ for all $\left(k^{m}, d, \ell\right) \gg 0$ and $\hat{f}\left(k^{m}, 0, \ell\right)=\hat{f}(0, d, \ell)=0$ (both the physical capital and the natural resource are essential in the production). Moreover, given $\left(\tilde{k}^{m}, \tilde{d}\right) \gg 0$, there exists a scalar $\tilde{\chi}$ such that $\left(d \hat{f}_{d}\left(k^{m}, d, 1\right)\right) /\left(\hat{f}_{\ell}\left(k^{m}, d, 1\right)\right) \geq \tilde{\chi}$ for $\left(k^{m}, d\right)$ satisfying $k^{m} \geq \tilde{k}^{m}$ and $0 \leq d \leq \tilde{d}$ (the ratio of the share of the resource to the share of labor is bounded away from zero when labor is fixed at unit level).

Assume that the labor force is constant and normalized to 1 . Write $f\left(k^{m}, d\right):=$ $\hat{f}\left(k^{m}, d, 1\right)$. Also assume that $f$ is strictly concave and $f_{k^{m}, d}\left(k^{m}, d\right) \gg 0$ for all $\left(k^{m}, d\right) \gg 0$. A consumption stream $\mathbf{x}=\left(x_{1}, x_{2}, \ldots\right)$ is feasible given initial stocks $\left(k_{1}^{m}, k_{1}^{n}\right) \gg 0$ if there exists a stream $\left\{\left(k_{2}^{m}, k_{2}^{n}\right),\left(k_{3}^{m}, k_{3}^{n}\right), \ldots\right\}$ such that

$$
x_{t}+k_{t+1}^{m} \leq f\left(k_{t}^{m}, k_{t}^{n}-k_{t+1}^{n}\right)+k_{t}^{m}, \quad x_{t} \geq 0, \quad k_{t}^{m} \geq 0, \quad k_{t}^{n} \geq 0
$$

for all $t \in \mathbb{N}$. Hence, production $f\left(k_{t}^{m}, k_{t}^{n}-k_{t+1}^{n}\right)$ is split between consumption $x_{t}$ and capital accumulation $k_{t+1}^{m}-k_{t}^{m}$ at each time $t$.

The assumptions made so far do not ensure that it is feasible to maintain a constant and positive consumption level forever. Therefore assume in addition 
that there exists from any $\left(k_{1}^{m}, k_{1}^{n}\right) \gg 0$ a constant stream with positive consumption. Cass and Mitra (1991) give a necessary and sufficient condition on $f$ for this assumption to hold. Under this additional assumption there exists an efficient constant consumption stream from any $\left(k_{1}^{m}, k_{1}^{n}\right) \gg 0$ (see Dasgupta and Mitra, 1983, Proposition 5). A technology satisfying the above assumptions is referred to as a Dasgupta-Heal-Solow (DHS) technology.

When establishing the implications of ERDU SWOs in the DHS growth model, it is a complication that production is increasing in capital only if resource extraction is positive, but a constant function of capital if resource extraction is zero. However, the analysis of the Ramsey model above can still be adapted to the DHS growth model.

Lemma 6. If $\mathbf{x} \in \mathbf{X}$ is feasible in a DHS technology, then $\overline{\mathbf{x}}$ is also feasible. If $\mathbf{x} \in \overline{\mathbf{X}}$ is feasible in a DHS technology, then $\mathbf{x}_{[]}$is also feasible.

Proof. These results follow as storage is costless in a DHS technology; cf. Asheim (1991, Lemma 4).

It follows from Lemma 2 of Asheim and Mitra (2010) and Lemma 6 above, combined with the concavity of $u$, that $\bar{W}(\mathbf{x})$ is finite for any feasible stream $\mathbf{x}$ in a DHS technology.

Denote by $x\left(k_{1}^{m}, k_{1}^{n}\right)$ the positive and constant level of consumption that can be sustained for ever along an efficient constant consumption stream from $\left(k_{1}^{m}, k_{1}^{n}\right) \gg 0$. It is possible to attach a sequence of shadow prices

$$
\left(p_{1}\left(k_{1}^{m}, k_{1}^{n}\right), p_{2}\left(k_{1}^{m}, k_{1}^{n}\right), \ldots, p_{t}\left(k_{1}^{m}, k_{1}^{n}\right), \ldots\right)
$$

to the corresponding stationary consumption stream (for a characterization of the prices, see Asheim and Mitra, 2010, Lemma 3). Write

$$
\beta_{\infty}\left(k_{1}^{m}, k_{1}^{n}\right)=\frac{\sum_{t=2}^{+\infty} p_{t}\left(k_{1}^{m}, k_{1}^{n}\right)}{\sum_{t=1}^{+\infty} p_{t}\left(k_{1}^{m}, k_{1}^{n}\right)}
$$

for the long-run discount factor at time 1 supporting this stationary stream.

Proposition 11. Consider an ERDU $S W F \succsim_{\beta, u}$ where $u$ is assumed to be strictly concave and continuously differentiable (on $\mathbb{R}_{++}$) with $\lim _{x \rightarrow 0} u^{\prime}(0)=0$, a Dasgupta-Heal-Solow technology, and initial stocks and resource $\left(k_{1}^{m}, k_{1}^{n}\right) \gg$ 0. There exists a unique optimal consumption stream, denoted $\mathbf{x}^{*}$, which is characterized as follows:

(a) If $\beta_{\infty}\left(k_{1}^{m}, k_{1}^{n}\right) \geq \beta$, then $\mathbf{x}^{*}$ is a stationary stream with $x_{t}^{*}=x\left(k_{1}^{m}, k_{1}^{n}\right)$ for all $t \geq 1$. 
(b) If $\beta_{\infty}\left(k_{1}^{m}, k_{1}^{n}\right)<\beta$, then $\mathbf{x}^{*}$ is a non-decreasing stream maximizing ( $1-$ $\beta) \sum_{t \in \mathbb{N}} \beta^{t-1} u\left(x_{t}\right)$ over all feasible and non-decreasing streams. The stream exhibits the following pattern:

- For $t<\tau, x_{t}^{*}<x_{t+1}^{*}$,

- For all $t \geq \tau, x_{t}^{*}=x\left(\left(k_{\tau}^{m}\right)^{*},\left(k_{\tau}^{n}\right)^{*}\right)$,

where $\tau:=\min \left\{t \in \mathbb{N} \mid \beta_{\infty}\left(\left(k_{t}^{m}\right)^{*},\left(k_{t}^{n}\right)^{*}\right) \geq \beta\right\}$.

The proof of Proposition 11 closely follows the proof of Proposition 10, differing only by substituting Lemma 6 for Lemma 5, Asheim and Mitra (2010, Lemma 6) for Asheim (1991, Proposition 6), and Asheim (1991, Lemma 4) for Asheim (1991, Lemma 3). Hence, it is not repeated here.

Proposition 11 shows that the consequences of a higher level of inequality aversion exhibited in the Ramsey growth model still hold in the Dasgupta-HealSolow model. Indeed:

- A more inequality averse RDU society $\succsim_{\beta, u}$ will prevent growth for a larger set of initial conditions than a less inequality averse society $\succsim_{\hat{\beta}, \hat{u}}$ (for $\left(k_{1}^{m}, k_{1}^{n}\right)$ satisfying $\beta_{\infty}\left(k_{1}^{m}, k_{1}^{n}\right) \geq \beta$ as opposed to $\left(k_{1}^{m}, k_{1}^{n}\right)$ satisfying $\left.\beta_{\infty}\left(k_{1}^{m}, k_{1}^{n}\right) \geq \hat{\beta}\right)$.

In particular, in the maximin case, growth is always prevented. Again, the maximin case represents an extreme form of inequality aversion, and less extreme degrees of inequality aversion may allow for growth in an initial phase.

\section{Conclusion}

The ERDU approach to intertemporal welfare has several appealing features. First, it offers a continuous and numerically representable criterion that reconciles intergenerational procedural equity and efficiency on the set of allocations that can be rearranged into non-decreasing streams. Second, compared to procedurally equitable, but incomplete, criteria like undiscounted utilitarianism and lexicographic maximin, it allows for more flexibility in the specification of inequality aversion. This is of particular importance in conflicts between the present generation and an infinite number of future generations (cf. Asheim, 2010). Last, it provides a consistent and intuitive interpretation of the ethical parameters determining the social discount rate. With the ERDU interpretation, we have obtained the provocative statement that inequality aversion increases the social discount rate along increasing consumption streams.

This statement is at odds with the traditional ethical approach to social discounting. It comes from the fact that ERDU criteria do satisfy procedural equity 
(the reason why people endorsing the traditional ethical approach have called for lower discount rates) while allowing for inequality-aversion-based discounting. We believe that ERDU may spark off new debates on social discounting within the ethical approach to social discounting.

The ERDU criterion can be operationalized. In particular, in benchmark growth models the ERDU optimal policies coincide with those promoted by the sustainable discounted utilitarian criterion that has been recently studied by Asheim and Mitra (2010). While its recommendations may not be new, the ERDU criterion offers an interesting new perspective that respects procedural equity and displays concerns for intergenerational redistribution. It sheds some new lights on what the present generation owes to future generations. On the one hand, we must guarantee that they will not be worse off than we are. On the other hand, intergenerational inequalities in favor of future generations should not be too large as this would be unfair to the present generation. This conception of intergenerational equity, more in line with the intuitive notion of distributional equity, may seem appealing to many.

\section{Appendix A}

Proof of Proposition 2. Assume that $\succsim$ satisfies axioms O, C, M, RD, RSEP, RSEF, RST and SA. Axioms O, C, and M imply that there exists a monotonic SWF $\bar{W}$ representing $\succsim$ on $\overline{\mathbf{X}}$. By axiom $\mathbf{S A}$, for all $\mathbf{x} \in \overline{\mathbf{X}}$, $\bar{W}(\mathbf{x})=\bar{W}\left(\mathbf{x}_{[]}\right)$. We can therefore restrict attention to the set $\mathbf{X}^{+}$.

Now, for each $T \in \mathbb{N}$, we introduce the following subset of $\mathbf{X}^{+}$:

$$
\left\{\mathbf{x} \in \mathbf{X}^{+}: x_{t}=x_{T+1}, \forall t \geq T+1\right\} \text {. }
$$

These are the nondecreasing intergenerational allocations with a constant tail from period $T+1$ onward. Denote the restriction of $\succsim_{\text {to }}$ this set by $\succsim_{T}$, which is a continuous monotonic weak order on the following rank-ordered set:

$$
\mathbf{X}_{T}^{+}=\left\{\left(x_{1}, \cdots, x_{T+1}\right) \in \mathbb{R}^{T+1}: x_{1} \leq \cdots \leq x_{T+1}\right\}
$$

Let $\mathcal{T}=\{1, \cdots, T+1\}$ denote the set of indices of the coordinates of $\mathbf{X}_{T}^{+}$.

We then proceed by showing that $\succsim_{T}$ satisfies a separability property. A subset of coordinates $\mathcal{S} \subset \mathcal{T}$ is said separable for $\succsim_{T}$ if for all $\left(x_{1}, \cdots, x_{T+1}\right)$, $\left(y_{1}, \cdots, y_{T+1}\right),\left(x_{1}^{\prime}, \cdots, x_{T+1}^{\prime}\right),\left(y_{1}^{\prime}, \cdots, y_{T+1}^{\prime}\right)$ in $\mathbf{X}_{T}^{+}$, if $x_{s}=x_{s}^{\prime}$ and $y_{s}=y_{s}^{\prime}$ for all $s \in \mathcal{S}$ and $x_{t}=y_{t}$ and $x_{t}^{\prime}=y_{t}^{\prime}$ for all $t \in \mathcal{T} \backslash \mathcal{S}$, then:

$$
\left(x_{1}, \cdots, x_{T+1}\right) \succsim_{T}\left(y_{1}, \cdots, y_{T+1}\right) \Longleftrightarrow\left(x_{1}^{\prime}, \cdots, x_{T+1}^{\prime}\right) \succsim_{T}\left(y_{1}^{\prime}, \cdots, y_{T+1}^{\prime}\right) .
$$

A subset of coordinates $\mathcal{S} \subset \mathcal{T}$ is said essential if there exist $\left(v_{s}\right)_{s \in \mathcal{S}}$ and $\left(w_{s}\right)_{s \in \mathcal{S}}$ 
in $\mathbb{R}^{|\mathcal{S}|}$ and $\left(z_{t}\right)_{t \in \mathcal{T} \backslash \mathcal{S}}$ in $\mathbb{R}^{|\mathcal{T} \backslash \mathcal{S}|}$ such that, if $\left(x_{1}, \cdots, x_{T+1}\right)$ and $\left(y_{1}, \cdots, y_{T+1}\right)$ are defined by $x_{s}=v_{s}$ and $y_{s}=w_{s}$ for all $s \in \mathcal{S}$ and $x_{t}=y_{t}=z_{t}$ for all $t \in \mathcal{T} \backslash \mathcal{S}$, then $\left(x_{1}, \cdots, x_{T+1}\right) \in \mathbf{X}_{T}^{+},\left(y_{1}, \cdots, y_{T+1}\right) \in \mathbf{X}_{T}^{+}$and $\left(x_{1}, \cdots, x_{T+1}\right) \succ_{T}$ $\left(y_{1}, \cdots, y_{T+1}\right)$. The set $\mathcal{T}$ is completely separable for the relation $\succsim_{T}$ if every subset $\mathcal{S} \subset \mathcal{T}$ is separable and essential.

To show that the set $\mathcal{T}=\{1, \cdots, T+1\}$ is completely separable for the ordering $\succsim_{T}$, we use Theorem 1 in Gorman $(1968)$. Let $(\mathcal{I}, \mathcal{K}, \mathcal{L}, \mathcal{M})$ be a partition of the set of indices $\mathcal{T}$ such that each subset is not the empty set. The theorem states that, If $\mathcal{I} \cup \mathcal{K}$ and $\mathcal{L} \cup \mathcal{K}$ are separable and essential for $\succsim_{T}$, then $\mathcal{I}, \mathcal{K}, \mathcal{L}, \mathcal{I} \cup \mathcal{L}$ and $\mathcal{I} \cup \mathcal{K} \cup \mathcal{L}$ are separable and essential for $\succsim_{T}$.

For any $t \leq T$, consider the following elements of $\mathbf{X}_{T}^{+}$:

$$
\begin{aligned}
& \left(z_{1}, \ldots, z_{t-1}, x_{t}, x_{t+1}, z_{t+2}, \ldots, z_{T+1}\right),\left(z_{1}, \ldots, z_{t-1}, y_{t}, y_{t+1}, z_{t+2}, \ldots, z_{T+1}\right), \\
& \left(w_{1}, \ldots, w_{t-1}, x_{t}, x_{t+1}, w_{t+2}, \ldots, w_{T+1}\right),\left(w_{1}, \ldots, w_{t-1}, y_{t}, y_{t+1}, w_{t+2}, \ldots, w_{T+1}\right) .
\end{aligned}
$$

By repeated application of axioms RSEF and RST, we have that:

$$
\begin{aligned}
& \left(z_{1}, \ldots, z_{t-1}, x_{t}, x_{t+1}, z_{t+2}, \ldots, z_{T+1}\right) \succsim_{T}\left(z_{1}, \ldots, z_{t-1}, y_{t}, y_{t+1}, z_{t+2}, \ldots, z_{T+1}\right) \\
& \Longleftrightarrow \\
& \left(x_{t}, x_{t+1}, z_{t+2}, \ldots, z_{T+1}\right) \succsim_{T-t+1}\left(y_{t}, y_{t+1}, z_{t+2}, \ldots, z_{T+1}\right) .
\end{aligned}
$$

By axiom RSEP,

$$
\begin{aligned}
& \left(x_{t}, x_{t+1}, z_{t+2}, \ldots, z_{T+1}\right) \succsim_{T-t+1}\left(y_{t}, y_{t+1}, z_{t+2}, \ldots, z_{T+1}\right) \\
& \Longleftrightarrow \\
& \left(x_{t}, x_{t+1}, w_{t+2}, \ldots, w_{T+1}\right) \succsim_{T-t+1}\left(y_{t}, y_{t+1}, w_{t+2}, \ldots, w_{T+1}\right) .
\end{aligned}
$$

And once again by repeated application of axioms RSEF and RST,

$$
\begin{aligned}
& \left(x_{t}, x_{t+1}, w_{t+2}, \ldots, w_{T+1}\right) \succsim_{T-t+1}\left(y_{t}, y_{t+1}, w_{t+2}, \ldots, w_{T+1}\right) \\
& \Longleftrightarrow \\
& \left(w_{1}, \ldots, w_{t-1}, x_{t}, x_{t+1}, w_{t+2}, \ldots, w_{T+1}\right) \succsim_{T}\left(w_{1}, \ldots, w_{t-1}, y_{t}, y_{t+1}, w_{t+2}, \ldots, w_{T+1}\right) .
\end{aligned}
$$

As a consequence,

$$
\begin{aligned}
& \left(z_{1}, \ldots, z_{t-1}, x_{t}, x_{t+1}, z_{t+2}, \ldots, z_{T+1}\right) \succsim_{T}\left(z_{1}, \ldots, z_{t-1}, y_{t}, y_{t+1}, z_{t+2}, \ldots, z_{T+1}\right) \\
& \Longleftrightarrow \\
& \left(w_{1}, \ldots, w_{t-1}, x_{t}, x_{t+1}, w_{t+2}, \ldots, w_{T+1}\right) \succsim_{T}\left(w_{1}, \ldots, w_{t-1}, y_{t}, y_{t+1}, w_{t+2}, \ldots, w_{T+1}\right),
\end{aligned}
$$

establishing that the set $\{t, t+1\}$ is separable for $\succsim_{T}$ for all $t \leq T$.

By RD and repeated application of RSEF and RST as above, the set $\{t, t+1\}$ is also essential.

Setting $\mathcal{I}=\{t\}, \mathcal{K}=\{t+1\}$ and $\mathcal{L}=\{t+2\}$, by Gorman's theorem the sets 
$\{t\},\{t+1\},\{t+2\},\{t, t+2\}$ and $\{t, t+1, t+2\}$ are separable and essential. Repeating the reasoning, all sets $\left\{t, t^{\prime}\right\} \subset \mathcal{T}$ are separable and essential. By unions of such sets and Gorman's theorem we can obtain any subset $\mathcal{S} \subset \mathcal{T}$. ${ }^{5}$ Hence $\mathcal{T}=\{1, \cdots, T+1\}$ is completely separable for the relation $\succsim_{T}$.

Because $\mathbf{X}_{T}^{+}$is a rank-ordered set and $\mathcal{T}=\{1, \cdots, T+1\}$ is completely separable for the relation $\succsim_{T}$ we know by Theorem 3.2 and Corollary 3.6 of Wakker (1993) that there exists a cardinal additive representation of $\succsim_{T}$ :

$$
\bar{W}_{T}(\mathbf{x})=\sum_{t=1}^{T} u_{t, T}\left(x_{t}\right)+V_{T}\left(x_{T+1}\right), \forall \mathbf{x} \in \mathbf{X}_{T}^{+}
$$

The functions $u_{t, T}$ and $V_{T}$ are all continuous and nondecreasing. In addition, by axioms M, RD, RSEF and RST, the functions $u_{1, T}$ and $V_{T}$ must be increasing. By cardinality, we may set $u_{t, T}(0)=0$ for all $t \leq T$ and $V_{T}(0)=0$ (normalization condition).

Now, representation (11) exists for $\succsim_{T}$ whatever $T \in \mathbb{N}$. Furthermore, $\succsim_{T}$ and $\succsim_{T+1}$ represent the same ordering on $\mathbf{X}_{T}^{+}$. By standard uniqueness results for additive functions on rank-ordered sets (Wakker, 1993), we can take after the appropriate normalization $u_{t, T} \equiv u_{t, T+1}$ and $V_{T} \equiv u_{T, T+1}+V_{T+1}$. We can henceforth drop the subscript $T$ in functions $u_{t, T}$.

By axioms RSEF and RST, we also know that $\bar{W}_{T}(\mathbf{x})=\sum_{t=1}^{T} u_{t}\left(x_{t}\right)+$ $V_{T}\left(x_{T+1}\right)$ and $\bar{W}_{T}(\mathbf{x})=\sum_{t=2}^{T+1} u_{t}\left(x_{t-1}\right)+V_{T+1}\left(x_{T+1}\right)$ represent the same preferences for all $\mathbf{x} \in \mathbf{X}_{T}^{+}$. By the cardinality of the additive representation and the normalization condition, there must exists a $\beta>0$ such that $u_{t+1}(x)=\beta u_{t}(x)$ and $V_{T+1}(x)=\beta V_{T}(x)$ for all $x \in \mathbb{R}_{+}$. Remark that $\beta$ does not depend on $t$. Denote $\bar{u} \equiv u_{1}$ and $V \equiv V_{1}$, we have the following representation of $\succsim_{T}$ :

$$
\bar{W}_{T}(\mathbf{x})=\sum_{t=1}^{T} \beta^{t-1} \bar{u}\left(x_{t}\right)+\beta^{T} V\left(x_{T+1}\right), \forall \mathbf{x} \in \mathbf{X}_{\mathbf{T}}^{+}
$$

with $\bar{u}$ and $V$ two increasing functions.

Now remark that we must also have $V(x)=\bar{u}(x)+\beta V(x)$, so that $V(x)=$ $\bar{u}(x) /(1-\beta)$. This implies that $\beta<1$ by axioms $\mathbf{M}$ and $\mathbf{R D}$. This also implies that $V(x)=\sum_{t=1}^{+\infty} \beta^{t-1} \bar{u}(x)$. Hence, we obtain the following representation of $\succsim$ on $\bigcup_{T \in \mathbb{N}} \mathbf{X}_{T}^{+}$by letting $u(x) \equiv \bar{u}(x) /(1-\beta)$ :

$$
\bar{W}(\mathbf{x})=(1-\beta) \sum_{t=1}^{+\infty} \beta^{t-1} u\left(x_{t}\right)
$$

Now it remains to prove that the representation extends to the whole set $\mathbf{X}^{+}$. For any $\mathbf{x} \in \mathbf{X}^{+}$, we define the sequence $\mathbf{x}^{1}, \mathbf{x}^{2}, \cdots, \mathbf{x}^{k}, \cdots$ of allocations in $\mathbf{X}^{+}$ as follows: for any $k \in \mathbb{N}, x_{t}^{k}=x_{t}$ for all $t \leq k$ and $x_{t}^{k}=x_{k}$ for all $t>k$. Each

\footnotetext{
${ }^{5}$ Singleton sets having been obtained in preceding steps.
} 
allocation in the sequence belongs to $\bigcup_{T \in \mathbb{N}} \mathbf{X}_{T}^{+}$, and $\lim _{k \rightarrow \infty} \sup _{t \in \mathbb{N}}\left|x_{t}^{k}-x_{t}\right|=0$, because we consider bounded streams. By $\mathbf{C}$, we obtain that $\bar{W}(\mathbf{x})=(1-$ $\beta) \sum_{t=1}^{+\infty} \beta^{t-1} u\left(x_{t}\right)$ is a SWF representing $\succsim$ on $\mathbf{X}^{+}$.

Proof of Lemma 3. We prove the result for the case where $T=0$. The extension to the case where $T>0$ (provided that $|L(\mathbf{x})| \geq T$ ) is straightforward: pull these dates out and do the arguments below on the remainder of the stream.

Hence, by (2) we seek to establish that, for all $\mathbf{x} \in \mathbf{X}$,

$$
\widetilde{W}(\mathbf{x}) \leq u(\ell(\mathbf{x}))
$$

Let $\mathbf{y}$ be defined by, $\forall t \in \mathbb{N}, y_{t}=\max \left\{x_{t}, \ell(\mathbf{x})\right\} \geq x_{t}$. By $\mathbf{M}, \widetilde{W}(\mathbf{x}) \leq \widetilde{W}(\mathbf{y})$. Hence, it is sufficient to show that $\tilde{W}(\mathbf{y}) \leq u(\ell(\mathbf{x}))$. Write $m:=\sup _{t} y_{t} ; m \in \mathbb{R}_{+}$ exists since $\mathbf{y}$ is bounded.

Case 1: $m=\ell(\mathbf{x})$. Then $y_{t}=\ell(\mathbf{x})$ for all $t$ and $\tilde{W}(\mathbf{y})=u(\ell(\mathbf{x}))$.

Case 2: $m>\ell(\mathbf{x})$. W.l.o.g. normalize the consumption scale s.t. $m=1$ and $\ell(\mathbf{x})=0$. By the definition of $m=1$ and $\ell(\mathbf{x})=0$, there exists

$$
\mathbf{z}=\left(1, \ldots, 1, \frac{1}{2}, 1, \ldots, 1, \frac{1}{3}, 1, \ldots, 1, \frac{1}{4}, 1, \ldots, 1, \frac{1}{5}, 1, \ldots, 1, \frac{1}{6}, 1, \ldots\right)
$$

such that, $\forall t \in \mathbb{N}, z_{t} \geq y_{t}$. By $\mathbf{M}, \widetilde{W}(\mathbf{x}) \leq \widetilde{W}(\mathbf{y}) \leq \widetilde{W}(\mathbf{z})$. Hence, it is sufficient to show that $\widetilde{W}(\mathbf{z}) \leq u(0)$.

By $\mathbf{S A}, \mathbf{z}$ is indifferent to each member of the following sequence of streams (where in $\mathbf{z}^{i}$ the 1 s appear at even dates $t$ satisfying $t \geq 2 i$ ):

$$
\begin{aligned}
\mathbf{z}^{1} & =\left(\frac{1}{2}, 1, \frac{1}{3}, 1, \frac{1}{4}, 1, \frac{1}{5}, 1, \frac{1}{6}, 1, \ldots\right) \\
\mathbf{z}^{2} & =\left(\frac{1}{3}, \frac{1}{2}, \frac{1}{4}, 1, \frac{1}{5}, 1, \frac{1}{6}, 1, \frac{1}{7}, 1, \ldots\right) \\
\mathbf{z}^{3} & =\left(\frac{1}{4}, \frac{1}{3}, \frac{1}{5}, \frac{1}{2}, \frac{1}{6}, 1, \frac{1}{7}, 1, \frac{1}{8}, 1, \ldots\right) \\
\mathbf{z}^{4} & =\left(\frac{1}{5}, \frac{1}{4}, \frac{1}{6}, \frac{1}{3}, \frac{1}{7}, \frac{1}{2}, \frac{1}{8}, 1, \frac{1}{9}, 1, \ldots\right) \\
& \ldots
\end{aligned}
$$

Write, $\forall i, j \in \mathbb{N}, m^{i j}:=\max _{t \in\{1, \ldots, j\}} z^{i}$. If $j \geq 2 i$, then $m^{i j}=1$. If there exists $k \in \mathbb{Z}_{+}$such that $j<2(i-k)$, then $m^{i j} \leq 1 /(2+k)$. Define, $\forall i, j \in \mathbb{N}$, $\mathbf{w}^{i j}$ by, $\forall t \in \mathbb{N}, w_{t}^{i j}=m^{i j}$ if $t \leq j$ and $w_{t}^{i j}=1$ otherwise.

Since, $\forall i, j \in \mathbb{N}, \mathbf{w}^{i j} \in \mathbf{X}^{+}$and, $\forall t \in \mathbb{N}, w_{t}^{i j} \geq z_{t}^{i}$, it follows, as $\succsim$ satisfies $\mathbf{M}$ and $\mathbf{S A}$ and is represented on $\overline{\mathbf{X}} \supseteq \mathbf{X}^{+}$by $\bar{W}$, that, for all $i, j \in \mathbb{N}$,

$$
\widetilde{W}(\mathbf{x}) \leq \widetilde{W}(\mathbf{y}) \leq \widetilde{W}(\mathbf{z})=\widetilde{W}\left(\mathbf{z}^{i}\right) \leq \widetilde{W}\left(\mathbf{w}^{i j}\right)=(1-\beta) \sum_{t \in \mathbb{N}} \beta^{t-1} u\left(w_{t}^{i j}\right) .
$$

Suppose $\widetilde{W}(\mathbf{z})=u(0)+\epsilon$, where $\epsilon>0$. Since $0<\beta<1$, one can choose $j \in \mathbb{N}$ such that $\beta^{j}(u(1)-u(0))<\frac{1}{2} \epsilon$. Since $u$ is continuous and, for fixed 
$j \in \mathbb{N}, m^{i j} \rightarrow 0$ as $i \rightarrow \infty$, one can choose $i \in \mathbb{N}$ such that $\left(1-\beta^{j}\right) u\left(m^{i j}\right)<$ $\left(1-\beta^{j}\right) u(0)+\frac{1}{2} \epsilon$. Then

$$
\widetilde{W}\left(\mathbf{w}^{i j}\right)=\left(1-\beta^{j}\right) u\left(m^{i j}\right)+\beta^{j} u(1)<u(0)+\frac{1}{2} \epsilon+\frac{1}{2} \epsilon=u(0)+\epsilon .
$$

This contradicts that $\widetilde{W}(\mathbf{z}) \leq \widetilde{W}\left(\mathbf{w}^{i j}\right)$ for all $i, j \in \mathbb{N}$. Hence, $\widetilde{W}(\mathbf{x}) \leq \widetilde{W}(\mathbf{y}) \leq$ $\widetilde{W}(\mathbf{z}) \leq u(0)=u(\ell(\mathbf{x}))$.

Proof of Lemma 4. Define $\mathbf{y}$ by, $\forall t \in \mathbb{N}, y_{t}=\ell(\mathbf{x})$. Construct a sequence of streams, $\mathbf{y}^{j}, j \in \mathbb{Z}_{+}$, inductively as follows: $\mathbf{y}^{0}=\mathbf{y}$ and, $\forall j \in \mathbb{N}$,

$$
\mathbf{y}^{j}= \begin{cases}\mathbf{y}^{j-1} & \text { if } x_{j} \geq \ell(\mathbf{x}) \\ \left(y_{1}^{j-1}, \ldots, y_{j-1}^{j-1}, x_{j},{ }_{j+1} \mathbf{y}^{j-1}\right) & \text { if } x_{j}<\ell(\mathbf{x})\end{cases}
$$

Note that, $\forall j \in \mathbb{N}, \ell\left(\mathbf{y}^{j}\right)=\ell(\mathbf{x})$. By Lemma $3, \forall j \in \mathbb{N}$,

$$
\widetilde{W}(\mathbf{x}) \leq \bar{W}\left(\mathbf{y}^{j}\right)
$$

Case 1: $|L(\mathbf{x})|<\infty$. In this case, there exists $j \in \mathbb{N}$ s.t., $\mathbf{y}^{j}=\overline{\mathbf{x}}$. As $\mathbf{y}^{j} \in \overline{\mathbf{X}}$, $\bar{W}\left(\mathbf{y}^{j}\right)=\widetilde{W}\left(\mathbf{y}^{j}\right) \leq \widetilde{W}(\mathbf{x})$ by $\mathbf{M}$. Hence, by $(4)$,

$$
\widetilde{W}(\mathbf{x})=\bar{W}\left(\mathbf{y}^{j}\right)=\bar{W}(\overline{\mathbf{x}})=W(\mathbf{x}) .
$$

Case 2: $|L(\mathbf{x})|=\infty$. Write, $\forall j \in \mathbb{N}, \ell^{j}:=\min _{t>j} x_{t}$. Note that $\ell^{j} \rightarrow \ell(\mathbf{x})$ as $j \rightarrow \infty$. For each $j \in \mathbb{N}$, define $\mathbf{z}^{j}$ by, $\forall t \in \mathbb{N}, z_{t}^{j}=\min \left\{x_{t}, \ell^{j}\right\}$. By $\mathbf{M}$ it holds, $\forall j \in \mathbb{N}$, that $\bar{W}\left(\mathbf{z}^{j}\right)=\widetilde{W}\left(\mathbf{z}^{j}\right) \leq \widetilde{W}(\mathbf{x})$, as $\mathbf{z}^{j} \in \overline{\mathbf{X}}$ and $\ell^{j}<\ell(\mathbf{x})$. Hence, by (12) it follows that, $\forall j \in \mathbb{N}$,

$$
\bar{W}\left(\mathbf{z}^{j}\right) \leq \widetilde{W}(\mathbf{x}) \leq \bar{W}\left(\mathbf{y}^{j}\right)
$$

For any $\epsilon>0$, we can choose $j \in \mathbb{N}$ s.t. $\bar{W}\left(\mathbf{y}^{j}\right)-\bar{W}\left(\mathbf{z}^{j}\right) \leq u(\ell(\mathbf{x}))-u\left(\ell^{j}\right)<\epsilon$ since $u$ is continuous and $\ell^{j} \rightarrow \ell(\mathbf{x})$ as $j \rightarrow \infty$. Combined with (13) and (4), this implies $\widetilde{W}(\mathbf{x})=\lim _{j \rightarrow \infty} \bar{W}\left(\mathbf{y}^{j}\right)=\bar{W}(\overline{\mathbf{x}})=W(\mathbf{x})$.

Proof of Proposition 6. If the ERDU SWO $\succsim_{\beta, u}$ satisfies axiom PDT, then $\beta \times \mathcal{C}_{u} \leq 1$. Assume that the ERDU SWO $\succsim_{\beta, u}$ satisfies axiom PDT. Consider $\mathbf{x} \in \mathbf{X}$ such that $x_{t}=0$ for all $t \leq \tau, x_{\tau} \leq x_{\tau+1}$, and $x_{t}>x_{\tau}+x_{\tau+1}$ for $t>\tau+1$. Now consider $\mathbf{y} \in \mathbf{X}$ such that $y_{\tau}+\varepsilon=x_{\tau} \leq x_{\tau+1}=y_{\tau+1}-\varepsilon$ and $y_{t}=x_{t}$ for all $t \neq \tau, \tau+1$, with $x_{\tau} \geq \varepsilon>0$. Since the ERDU SWO $\succsim_{\beta, u}$ satisfies axiom PDT, it follows from the representation (3) of ERDU SWOs that:

$$
\beta^{\tau-1} u\left(x_{\tau}\right)+\beta^{\tau} u\left(x_{\tau+1}\right) \geq \beta^{\tau-1} u\left(y_{\tau}\right)+\beta^{\tau} u\left(y_{\tau+1}\right)=\beta^{\tau-1} u\left(x_{\tau}-\varepsilon\right)+\beta^{\tau} u\left(x_{\tau+1}+\varepsilon\right) .
$$


This inequality can be rewritten:

$$
1 \geq \beta \frac{u\left(x_{\tau^{\prime}}+\varepsilon\right)-u\left(x_{\tau^{\prime}}\right)}{u\left(x_{\tau}\right)-u\left(x_{\tau}-\varepsilon\right)} .
$$

The construction of allocations $\mathbf{x}$ and $\mathbf{y}$ yielding this inequality can be done for any two integers $\tau<\tau^{\prime}$, and for any real numbers $0<\varepsilon \leq x_{\tau} \leq x_{\tau^{\prime}}$. Hence,

$$
1 \geq \sup _{0<\varepsilon \leq x \leq x^{\prime}} \beta \times \frac{u\left(x^{\prime}+\varepsilon\right)-u\left(x^{\prime}\right)}{u(x)-u(x-\varepsilon)}=\beta \times \mathcal{C}_{u}
$$

If $\beta \times \mathcal{C}_{u} \leq 1$ holds for the ERDU SWO $\succsim_{\beta, u}$, then $\succsim_{\beta, u}$ satisfies axiom $\boldsymbol{P D T}$. Assume that $1 \geq \beta \times \mathcal{C}_{u}$ holds for the ERDU SWO $\succsim_{\beta, u}$. Consider $\mathbf{x}$, $\mathbf{y} \in \mathbf{X}$ such that $\varepsilon \leq y_{\tau}+\varepsilon=x_{\tau} \leq x_{\tau^{\prime}}=y_{\tau^{\prime}}-\varepsilon$, and $y_{t}=x_{t}$ for all $t \neq \tau, \tau^{\prime}$, where $x_{\tau} \geq \varepsilon>0$ and $\tau, \tau^{\prime} \in \mathbb{N}$. We want to show that $\mathbf{x} \succsim_{\beta, u} \mathbf{y}$.

If $\ell(\mathbf{x}) \leq x_{\tau^{\prime}}$, then $\mathbf{x} \succsim_{\beta, u} \mathbf{y}$ by a dominance argument for those components that matter. In the case where $x_{\tau^{\prime}}<\ell(\mathbf{x})<y_{\tau^{\prime}}$, the same argument as below applies, replacing $y_{\tau^{\prime}}$ by $\ell(\mathbf{x})$. Therefore, assume that $y_{\tau^{\prime}} \leq \ell(\mathbf{x})$.

Using representation (3), it follows that $\mathbf{x} \succsim_{\beta, u} \mathbf{y}$ if and only if:

$$
W(\mathbf{x})-W(\mathbf{y})=\sum_{\bar{r}_{\tau}(\mathbf{y}) \leq r \leq r_{\tau}(\mathbf{x})} \beta^{r-1}\left(u\left(x_{[r]}\right)-u\left(y_{[r]}\right)\right)-\sum_{\bar{r}_{\tau^{\prime}}(\mathbf{x}) \leq r \leq r_{\tau^{\prime}}(\mathbf{y})} \beta^{r-1}\left(u\left(y_{[r]}\right)-u\left(x_{[r]}\right)\right) \geq 0 .
$$

For $\bar{r}_{\tau}(\mathbf{y}) \leq r \leq r_{\tau}(\mathbf{x})$, we have $u\left(x_{[r]}\right)-u\left(y_{[r]}\right) \geq 0$, and for $\bar{r}_{\tau^{\prime}}(\mathbf{x}) \leq r \leq$ $r_{\tau^{\prime}}(\mathbf{y})$, we have $u\left(y_{[r]}\right)-u\left(x_{[r]}\right) \geq 0$. Hence:

$$
\begin{gathered}
\sum_{\bar{r}_{\tau}(\mathbf{y}) \leq r \leq r_{\tau}(\mathbf{x})} \beta^{r-1}\left(u\left(x_{[r]}\right)-u\left(y_{[r]}\right)\right)-\sum_{\bar{r}_{\tau^{\prime}}(\mathbf{x}) \leq r \leq r_{\tau^{\prime}}(\mathbf{y})} \beta^{r-1}\left(u\left(y_{[r]}\right)-u\left(x_{[r]}\right)\right) \\
\geq \beta^{r_{\tau}(\mathbf{x})-1} \times \sum_{\bar{r}_{\tau}(\mathbf{y}) \leq r \leq r_{\tau}(\mathbf{x})}\left(u\left(x_{[r]}\right)-u\left(y_{[r]}\right)\right)-\beta^{\bar{r}_{\tau^{\prime}}(\mathbf{x})-1} \times \sum_{\bar{r}_{\tau^{\prime}}(\mathbf{x}) \leq r \leq r_{\tau^{\prime}}(\mathbf{y})}\left(u\left(y_{[r]}\right)-u\left(x_{[r]}\right)\right) .
\end{gathered}
$$

By definition of the Pigou-Dalton transfer, $\sum_{\bar{r}_{\tau}(\mathbf{y}) \leq r \leq r_{\tau}(\mathbf{x})}\left(u\left(x_{[r]}\right)-u\left(y_{[r]}\right)\right)=$ $u\left(x_{\tau}\right)-u\left(y_{\tau}\right)$ and $\sum_{\bar{r}_{\tau^{\prime}}(\mathbf{x}) \leq r \leq r_{\tau^{\prime}}(\mathbf{y})}\left(u\left(y_{[r]}\right)-u\left(x_{[r]}\right)\right)=u\left(y_{\tau^{\prime}}\right)-u\left(x_{\tau^{\prime}}\right)$. Therefore:

$$
\begin{aligned}
W(\mathbf{x})-W(\mathbf{y}) & \geq \beta^{r_{\tau}(\mathbf{x})-1}\left(u\left(x_{\tau}\right)-u\left(y_{\tau}\right)\right)-\beta^{\bar{r}_{\tau^{\prime}}(\mathbf{x})-1}\left(u\left(y_{\tau^{\prime}}\right)-u\left(x_{\tau^{\prime}}\right)\right) \\
& =\beta^{r_{\tau}(\mathbf{x})-1}\left(u\left(x_{\tau}\right)-u\left(y_{\tau}\right)\right)\left(1-\beta^{\bar{r}_{\tau^{\prime}}(\mathbf{x})-r_{\tau}(\mathbf{x})} \frac{u\left(y_{\tau^{\prime}}\right)-u\left(x_{\tau^{\prime}}\right)}{u\left(x_{\tau}\right)-u\left(y_{\tau}\right)}\right) \\
& \geq \beta^{r_{\tau}(\mathbf{x})-1}\left(u\left(x_{\tau}\right)-u\left(y_{\tau}\right)\right)\left(1-\beta \times \frac{u\left(x_{\tau^{\prime}}+\varepsilon\right)-u\left(x_{\tau^{\prime}}\right)}{u\left(x_{\tau}\right)-u\left(x_{\tau}-\varepsilon\right)}\right) .
\end{aligned}
$$

Since $1 \geq \beta \times \mathcal{C}_{u}$, it follows that $W(\mathbf{x})-W(\mathbf{y}) \geq 0$ and thus $\mathbf{x} \succsim_{\beta, u} \mathbf{y}$. 
The next lemma is needed for the proof of Proposition 7. Write

$$
\begin{gathered}
\hat{X}=\left\{\left(x_{1}, x_{2}, x_{3}, x_{4}\right) \in \mathbb{R}_{+}^{4}: 0 \leq x_{1}<x_{2} \leq x_{3}<x_{4},\right. \text { where } \\
\left.x_{4}=\hat{u}^{-1}\left(\hat{u}\left(x_{3}\right)+\hat{\beta}^{\tau-\tau^{\prime}}\left(\hat{u}\left(x_{2}\right)-\hat{u}\left(x_{1}\right)\right)\right) \text { for some } \tau, \tau^{\prime} \in \mathbb{N} \text { with } \tau<\tau^{\prime}\right\}, \\
\widetilde{\mathcal{C}}_{u, \hat{u}}=\sup _{\left(x_{1}, x_{2}, x_{3}, x_{4}\right) \in \hat{X}} \frac{\left[u\left(x_{4}\right)-u\left(x_{3}\right)\right] /\left[\hat{u}\left(x_{4}\right)-\hat{u}\left(x_{3}\right)\right]}{\left[u\left(x_{2}\right)-u\left(x_{1}\right)\right] /\left[\hat{u}\left(x_{2}\right)-\hat{u}\left(x_{1}\right)\right]} .
\end{gathered}
$$

Lemma 7. $\mathcal{C}_{u, \hat{u}}=\widetilde{\mathcal{C}}_{u, \hat{u}}$.

Proof. Write $y_{1}=\hat{u}\left(x_{1}\right), y_{2}=\hat{u}\left(x_{2}\right), y_{3}=\hat{u}\left(x_{3}\right)$ and $y_{4}=\hat{u}\left(x_{4}\right)$. Then

$$
\mathcal{C}_{u, \hat{u}}=\sup _{0 \leq y_{1}<y_{2} \leq y_{3}<y_{4}} \frac{\left[u \circ \hat{u}^{-1}\left(y_{4}\right)-u \circ \hat{u}^{-1}\left(y_{3}\right)\right] /\left[y_{4}-y_{3}\right]}{\left[u \circ \hat{u}^{-1}\left(y_{2}\right)-u \circ \hat{u}^{-1}\left(y_{1}\right)\right] /\left[y_{2}-y_{1}\right]}=G_{u \circ \hat{u}^{-1}},
$$

where $G_{u \circ \hat{u}^{-1}}$ is Chateauneuf, Cohen and Meilijson's (2005) 'greediness' index for the function $u \circ \hat{u}^{-1}$. Write also

$$
\begin{aligned}
& \hat{X}_{\lambda}=\left\{\left(y_{1}, y_{2}, y_{3}, y_{4}\right) \in \mathbb{R}_{+}^{4}: 0 \leq y_{1}<y_{2} \leq y_{3}<y_{4}, \text { where } \frac{y_{4}-y_{3}}{y_{2}-y_{1}}=\lambda\right\}, \\
& G_{u \circ \hat{u}^{-1}}(\lambda)=\sup _{\left(y_{1}, y_{2}, y_{3}, y_{4}\right) \in \hat{X}_{\lambda}} \frac{\left[u \circ \hat{u}^{-1}\left(y_{4}\right)-u \circ \hat{u}^{-1}\left(y_{3}\right)\right] /\left[y_{4}-y_{3}\right]}{\left[u \circ \hat{u}^{-1}\left(y_{2}\right)-u \circ \hat{u}^{-1}\left(y_{1}\right)\right] /\left[y_{2}-y_{1}\right]} .
\end{aligned}
$$

Then $\widetilde{\mathcal{C}}_{u, \hat{u}}=\sup _{\lambda=\hat{\beta}^{\tau-\tau^{\prime}}, \tau<\tau^{\prime}} G_{u \circ \hat{u}^{-1}}(\lambda)$. By Chateauneuf, Cohen and Meilijson (2005, Lemma 1), $G_{u \circ \hat{u}^{-1}}=G_{u \circ \hat{u}^{-1}}(\lambda)$ for any $\lambda>0$, so that $\mathcal{C}_{u, \hat{u}}=\widetilde{\mathcal{C}}_{u, \hat{u}}$.

Proof of Proposition 7. If $\succsim_{\beta, u}$ is at least as inequality averse as $\succsim_{\hat{\beta}, \hat{u}}$, then $\mathcal{D}_{\beta, \hat{\beta}} \geq \mathcal{C}_{u, \hat{u}}$. Assume that $\succsim_{\beta, u}$ is at least as inequality averse as $\succsim_{\hat{\beta}, \hat{u}}$. Consider $\mathbf{x}, \mathbf{y} \in \mathbf{X}$ such that, for some $\tau, \tau^{\prime} \in \mathbb{N}$ with $\tau<\tau^{\prime}$ :

- $x_{t}=y_{t}=0$ for all $t<\tau$;

- $0 \leq y_{\tau}<x_{\tau} \leq y_{t}=x_{t} \leq x_{\tau^{\prime}}$ for all $\tau<t<\tau^{\prime}$;

- $y_{\tau^{\prime}}=\hat{u}^{-1}\left(\hat{u}\left(x_{\tau^{\prime}}\right)+\hat{\beta}^{\tau-\tau^{\prime}}\left(\hat{u}\left(x_{\tau}\right)-\hat{u}\left(y_{\tau}\right)\right)\right)$ so that $y_{\tau^{\prime}} \geq x_{\tau^{\prime}}$;

- $y_{t}=x_{t}>y_{\tau}$ for $t>\tau^{\prime}$.

(a) By construction, $\hat{\beta}^{\tau^{\prime}}\left(\hat{u}\left(y_{\tau^{\prime}}\right)-\hat{u}\left(x_{\tau^{\prime}}\right)\right)=\hat{\beta}^{\tau}\left(\hat{u}\left(x_{\tau}\right)-\hat{u}\left(y_{\tau}\right)\right)$, so that $\mathbf{x} \sim_{\hat{\beta}, \hat{u}} \mathbf{y}$. (b) Because $\mathbf{y} \succ_{I} \mathbf{x}$ and $\succsim_{\beta, u}$ is at least as inequality averse as $\succsim_{\hat{\beta}, \hat{u}}, \mathbf{x} \succsim_{\beta, u} \mathbf{y}$ so that $\beta^{\tau^{\prime}}\left(u\left(y_{\tau^{\prime}}\right)-u\left(x_{\tau^{\prime}}\right)\right) \leq \beta^{\tau}\left(u\left(x_{\tau}\right)-u\left(y_{\tau}\right)\right)$. Facts (a) and (b) imply

$$
\frac{\beta^{\tau} / \hat{\beta}^{\tau}}{\beta^{\tau^{\prime}} / \hat{\beta}^{\tau^{\prime}}} \geq \frac{\left(u\left(y_{\tau^{\prime}}\right)-u\left(x_{\tau^{\prime}}\right)\right) /\left(\hat{u}\left(y_{\tau^{\prime}}\right)-\hat{u}\left(x_{\tau^{\prime}}\right)\right)}{\left(u\left(x_{\tau}\right)-u\left(y_{\tau}\right)\right) /\left(\hat{u}\left(x_{\tau}\right)-\hat{u}\left(y_{\tau}\right)\right)} .
$$


The construction of $\mathbf{x}$ and $\mathbf{y}$ yielding this inequality can be done for any two integers $\tau<\tau^{\prime}$, and for any real numbers $0 \leq y_{\tau}<x_{\tau} \leq x_{\tau^{\prime}}<y_{\tau^{\prime}}$ such that $y_{\tau^{\prime}}=\hat{u}^{-1}\left(\hat{u}\left(x_{\tau^{\prime}}\right)+\hat{\beta}^{\tau-\tau^{\prime}}\left(\hat{u}\left(x_{\tau}\right)-\hat{u}\left(y_{\tau}\right)\right)\right)$. Then

$$
\mathcal{D}_{\beta, \hat{\beta}}=\inf _{t<t^{\prime}} \frac{\beta^{\tau} / \hat{\beta}^{\tau}}{\beta^{\tau^{\prime}} / \hat{\beta}^{\tau^{\prime}}} \geq \sup _{\left(x_{1}, x_{2}, x_{3}, x_{4}\right) \in \hat{X}} \frac{\left[u\left(x_{4}\right)-u\left(x_{3}\right)\right] /\left[\hat{u}\left(x_{4}\right)-\hat{u}\left(x_{3}\right)\right]}{\left[u\left(x_{2}\right)-u\left(x_{1}\right)\right] /\left[\hat{u}\left(x_{2}\right)-\hat{u}\left(x_{1}\right)\right]}=\widetilde{\mathcal{C}_{u, \hat{u}}} .
$$

By Lemma 7 it follows that $\mathcal{D}_{\beta, \hat{\beta}} \geq \mathcal{C}_{u, \hat{u}}$.

If $\mathcal{D}_{\beta, \hat{\beta}} \geq \mathcal{C}_{u, \hat{u}}$, then $\succsim_{\beta, u}$ is at least as inequality averse as $\succsim_{\hat{\beta}, \hat{u}}$. Assume that $\mathbf{y} \succ_{I} \mathbf{x}$ and $\mathbf{x} \sim_{\hat{\beta}, \hat{u}} \mathbf{y}{ }^{6}{ }^{6}$ We want to show that $\mathbf{x} \succsim_{\beta, u} \mathbf{y}$ if $\mathcal{D}_{\beta, \hat{\beta}} \geq \mathcal{C}_{u, \hat{u}}$.

If $\ell(\mathbf{x}) \leq x_{\tau^{\prime}}$, then $\mathbf{x} \succsim_{\beta, u} \mathbf{y}$ and $\mathbf{x} \succsim_{\hat{\beta}, \hat{u}} \mathbf{y}$. If $x_{\tau^{\prime}}<\ell(\mathbf{x})<y_{\tau^{\prime}}$, then the argument below applies, replacing $y_{\tau^{\prime}}$ by $\ell(\mathbf{x})$. Therefore, assume that $y_{\tau^{\prime}} \leq \ell(\mathbf{x})$.

By using $\hat{\beta}^{r_{\tau}(\mathbf{x})}\left(\hat{u}\left(x_{\tau}\right)-\hat{u}\left(y_{\tau}\right)\right)=\hat{\beta}^{\bar{r}_{\tau^{\prime}}(\mathbf{x})}\left(\hat{u}\left(y_{\tau^{\prime}}\right)-\hat{u}\left(x_{\tau^{\prime}}\right)\right)$ (which follows from Eq. (3) and $\mathbf{x} \sim_{\hat{\beta}, \hat{u}} \mathbf{y}$ ) we obtain

$$
\begin{aligned}
& \beta^{r_{\tau}(\mathbf{x})}\left(u\left(x_{\tau}\right)-u\left(y_{\tau}\right)\right)-\beta^{\bar{r}_{\tau^{\prime}}(\mathbf{x})}\left(u\left(y_{\tau^{\prime}}\right)-u\left(x_{\tau^{\prime}}\right)\right) \\
& =\hat{\beta}^{\bar{r}_{\tau^{\prime}}(\mathbf{x})}\left(\hat{u}\left(y_{\tau^{\prime}}\right)-\hat{u}\left(x_{\tau^{\prime}}\right)\right)\left(\frac{\beta^{r_{\tau}(\mathbf{x})}\left(u\left(x_{\tau}\right)-u\left(y_{\tau}\right)\right)}{\hat{\beta}^{r_{\tau}(\mathbf{x})}\left(\hat{u}\left(x_{\tau}\right)-\hat{u}\left(y_{\tau}\right)\right)}-\frac{\beta^{\bar{r}_{\tau^{\prime}}(\mathbf{x})}\left(u\left(y_{\tau^{\prime}}\right)-u\left(x_{\tau^{\prime}}\right)\right)}{\hat{\beta}^{\bar{r}_{\tau^{\prime}}(\mathbf{x})}\left(\hat{u}\left(y_{\tau^{\prime}}\right)-\hat{u}\left(x_{\tau^{\prime}}\right)\right)}\right) \\
& =\frac{\beta^{\bar{r}_{\tau^{\prime}}(\mathbf{x})}\left(\hat{u}\left(y_{\tau^{\prime}}\right)-\hat{u}\left(x_{\tau^{\prime}}\right)\right)}{\frac{\hat{u}\left(x_{\tau}\right)-\hat{u}\left(y_{\tau}\right)}{u\left(x_{\tau}\right)-u\left(y_{\tau}\right)}}\left(\left(\frac{\beta}{\hat{\beta}}\right)^{r_{\tau}(\mathbf{x})-\bar{r}_{\tau^{\prime}}(\mathbf{x})}-\frac{\frac{u\left(y_{\tau^{\prime}}\right)-u\left(x_{\tau^{\prime}}\right)}{\hat{u}\left(y_{\tau^{\prime}}\right)-\hat{u}\left(x_{\tau^{\prime}}\right)}}{\frac{u\left(x_{\tau}\right)-u\left(y_{\tau^{\prime}}\right)}{\hat{u}\left(x_{\tau}\right)-\hat{u}\left(y_{\tau}\right)}}\right) \\
& \geq \frac{\beta^{\bar{r}_{\tau^{\prime}}(\mathbf{x})}\left(\hat{u}\left(y_{\tau^{\prime}}\right)-\hat{u}\left(x_{\tau^{\prime}}\right)\right)}{\frac{\hat{u}\left(x_{\tau}\right)-\hat{u}\left(y_{\tau}\right)}{u\left(x_{\tau}\right)-u\left(y_{\tau}\right)}}\left(\mathcal{D}_{\beta, \hat{\beta}}-\mathcal{C}_{u, \hat{u}}\right) .
\end{aligned}
$$

It now follows from Eq. (3) that that $\mathbf{x} \succsim_{\beta, u} \mathbf{y}$ whenever $\mathcal{D}_{\beta, \hat{\beta}} \geq \mathcal{C}_{u, \hat{u}}$.

\section{References}

Asheim, G.B. (1991). "Unjust intergenerational allocations", Journal of Economic Theory, 54, 350-371.

Asheim, G.B. (2010), "Intergenerational equity", Annual Review of Economics, 2 (2010), 197-222.

Asheim, G.B., Buchholz, W. (2003). "The malleability of undiscounted utilitarianism as a criterion of intergenerational justice", Economica, 70, 405-422.

\footnotetext{
${ }^{6}$ If $\mathbf{x} \succ_{\hat{\beta}, \hat{u}} \mathbf{y}$, then by monotonicity there exists $x_{\tau}-y_{\tau}>\varepsilon>0$ such that $\mathbf{x}^{\prime}$ defined by $x_{\tau}^{\prime}=x_{\tau}-\varepsilon$ (where $\tau$ is as in Definition 4) and $x_{t}^{\prime}=x_{t}$ for all $t \neq \tau$ satisfies $\mathbf{x}^{\prime} \sim_{\hat{\beta}, \hat{u}} \mathbf{y}$. By monotonicity, $\mathbf{x} \succ_{\beta, u} \mathbf{x}^{\prime}$, by transitivity, $\mathbf{x} \succ_{\beta, u} \mathbf{y}$, while it is also the case that $\mathbf{y} \succ_{I} \mathbf{x}^{\prime}$. Hence, if $\left(\mathbf{y} \succ_{I} \mathbf{x} \& \mathbf{x} \sim_{\hat{\beta}, \hat{u}} \mathbf{y}\right) \Longrightarrow\left(\mathbf{x} \succsim_{\beta, u} \mathbf{y}\right)$ then $\left(\mathbf{y} \succ_{I} \mathbf{x} \& \mathbf{x} \succ_{\hat{\beta}, \hat{u}} \mathbf{y}\right) \Longrightarrow\left(\mathbf{x} \succ_{\beta, u} \mathbf{y}\right)$.
} 
Asheim, G.B., Buchholz, W., Tungodden, B. (2001). "Justifying sustainability", Journal of Environmental Economics and Management, 41, 252-268.

Asheim, G.B., Mitra, T. (2010). "Sustainability and discounted utilitarianism in models of economic growth", Mathematical Social Sciences, 59, 148-169.

Asheim, G.B., Mitra, T., Tungodden, B. (2010). "Sustainable recursive social welfare functions", forthcoming in Economic Theory.

Atkinson, A.B. (1970). "On the measurement of inequality", Journal of Economic Theory, 2, 244-263.

Basu, K., Mitra, T. (2003). "Aggregating infinite utility streams with intergenerational equity: the impossibility of being Paretian", Econometrica, 71, 1557-1563.

Basu, K., Mitra, T. (2007). "Utilitarianism for infinite utility streams: A new welfare criterion and its axiomatic characterization", Journal of Economic Theory, 133, $350-373$.

Birchenhall, C.R., Grout, P. (1979). "On equal plans with infinite horizon", Journal of Economic Theory, 21, 249-264.

Bleichrodt, H., Rhode, K.I.M., Wakker, P.P. (2008). "Ordering infinite utility streams", Journal of Mathematical Psychology, 52, 341-347.

Bossert, W. (1990). "An axiomatization of the single-series Ginis", Journal of Economic Theory 50, 82-92.

Bossert, W., Sprumont, Y., Suzumura, K. (2007). "Ordering infinite utility streams", Journal of Economic Theory, 135, 579-589.

Cass, D., Mitra, T. (1991). "Indefinitely sustained consumption despite exhaustible natural resources", Economic Theory, 1, 119-146.

Chateauneuf, A., Cohen, M., Meilijson, I. (2005). "More pessimism than greediness: a characterization of monotone risk aversion in the rank-dependent expected utility model", Economic Theory, 25, 649-667.

Chichilnisky, G. (1996). "An axiomatic approach to sustainable development", Social Choice and Welfare, 13, 231-257.

Dasgupta, P.S. (2008). "Discounting climate change", Journal of Risk and Uncertainty, 37, 141-169.

Dasgupta, P.S., Heal, G. (1974). "The optimal depletion of exhaustible resources", The Review of Economic Studies, 41, Symposium on the Economics of Exhaustible Resources, 3-28. 
Dasgupta, S., Mitra, T. (1983). "Intergenerational equity and efficient allocation of exhaustible resources", International Economic Review, 24, 133-153.

Diamond, P. (1965). "The evaluation of infinite utility streams", Econometrica, 33, $170-177$.

Donaldson, D., Weymark, J.A. (1980). "A single-parameter generalization of the Gini indices of inequality", Journal of Economic Theory, 22, 67-86.

Donaldson, D., Weymark, J.A. (1983). "Ethically flexible Gini indices for income distributions in the continuum", Journal of Economic Theory 29, 353-358.

Ebert, U. (1988). "Measurement of inequality: An attempt at unification and generalization", Social Choice and Welfare, 5, 147-169.

Fleurbaey, M., Michel, P. (2003). "Intertemporal equity and the extension of the Ramsey criterion", Journal of Mathematical Economics, 39, 777-802.

Gilboa, I. (1987). "Expected utility with purely subjective non-additive probabilities", Journal of Mathematical Economics, 16, 65-88.

Gorman, W.G. (1968). "The structure of utility functions", Review of Economic Studies, 35, 367-390.

Grant, S., Quiggin, J. (2005). "Increasing uncertainty: a definition", Mathematical Social Sciences, 49, 117-141.

Hara, C., Shinotsuka, T., Suzumura, K., Xu, Y. (2008). "Continuity and egalitarianism in the evaluation of infinite streams", Social Choice and Welfare, 31, 179-191.

Harrod, R.F. (1948). Towards a Dynamic Economy: Some Recent Developments of Economic Theory and their Application to Policy, London: Macmillan.

Koopmans, T.C. (1960). "Stationary ordinal utility and impatience", Econometrica, $28,287-309$.

Lauwers, L. (2010). "Ordering infinite utility streams comes at the cost of a nonRamsey set", Journal of Mathematical Economics, 46, 32-37.

Mirrlees, J.A. (1967). "Optimum growth when technology is changing", The Review of Economic Studies, 34, 95-124.

Nordhaus, W.D. (2007). "The Stern Review on the economics of climate change", Journal of Economic Literature, 45, 687-702.

Nordhaus, W.D. (2008). A Question of Balance: Weighing the Options on Global Warming Policies, New Haven, CT: Yale University Press. 
Pigou, A.C. (1920). The Economics of Welfare, London: Macmillan.

Ramsey, F.P. (1928). "A mathematical theory of saving", The Economic Journal, 38, 543-559.

Rawls, J. (1999). A Theory of Justice, revised edition, Cambridge, MA: The Belknap Press of the Harvard University Press.

Rébillé, Y. (2007). "Patience in some non-additive models", Journal of Mathematical Economics, 43, 749-763.

Solow, R.M. (1974). "Intergenerational Equity and Exhaustible Resources", The Review of Economic Studies, Symposium on the economics of exhaustible resources, $29-45$.

Stern, N.H. (2006). The Stern Review of the Economics of Climate Change, Cambridge, UK: Cambridge University Press.

Svensson, L.-G. (1980). "Equity among generations", Econometrica, 48, 1251-1256.

Van Liedekerke, L., Lauwers L. (1997). "Sacrificing the patrol: Utilitarianism, future generations and infinity", Economics and Philosophy 13, 159-74.

Wakker, P.P. (1993). "Additive representations on rank-ordered sets II. The topological approach", Journal of Mathematical Economics, 22, 1-26.

Weitzman, M.L. (2007). "The Stern Review of the economics of climate change", Journal of Economic Literature, 45, 703-724.

Weymark, J. (1981). "Generalized Gini inequality indices", Mathematical Social Sciences, 1, 409-430.

Zame, W.R. (2007). "Can intergenerational equity be operationalized?", Theoretical Economics, 2, 187-202. 


\section{CESifo Working Paper Series}

for full list see www.cesifo-group.org/wp

(address: Poschingerstr. 5, 81679 Munich, Germany, office@cesifo.de)

3132 John Komlos and Marek Brabec, The Trend of BMI Values by Centiles of US Adults, Birth Cohorts 1882-1986, July 2010

3133 Emin Karagözoğlu and Arno Riedl, Information, Uncertainty, and Subjective Entitlements in Bargaining, July 2010

3134 John Boyd, Gianni De Nicolò and Elena Loukoianova, Banking Crises and Crisis Dating: Theory and Evidence, July 2010

3135 Michael R. Baye, Dan Kovenock and Casper G. de Vries, The Herodotus Paradox, July 2010

3136 Martin Kolmar and Hendrik Rommeswinkel, Group Contests with Complementarities in Efforts, July 2010

3137 Carolina Manzano and Xavier Vives, Public and Private Learning from Prices, Strategic Substitutability and Complementarity, and Equilibrium Multiplicity, July 2010

3138 Axel Löffler, Gunther Schnabl and Franziska Schobert, Inflation Targeting by Debtor Central Banks in Emerging Market Economies, July 2010

3139 Yu-Fu Chen and Michael Funke, Global Warming and Extreme Events: Rethinking the Timing and Intensity of Environmental Policy, July 2010

3140 Lawrence M. Kahn, Labor Market Policy: A Comparative View on the Costs and Benefits of Labor Market Flexibility, July 2010

3141 Ben J. Heijdra, Jochen O. Mierau and Laurie S.M. Reijnders, The Tragedy of Annuitization, July 2010

3142 Erkki Koskela, Outsourcing Cost and Tax Progression under Nash Wage Bargaining with Flexible Outsourcing, July 2010

3143 Daniel Osberghaus and Christiane Reif, Total Costs and Budgetary Effects of Adaptation to Climate Change: An Assessment for the European Union, August 2010

3144 Philip E. Graves, Benefit-Cost Analysis of Environmental Projects: A Plethora of Systematic Biases, August 2010

3145 Sabrina Di Addario and Daniela Vuri, Entrepreneurship and Market Size. The Case of Young College Graduates in Italy, August 2010

3146 Shoshana Amyra Grossbard and Alfredo Marvăo Pereira, Will Women Save more than Men? A Theoretical Model of Savings and Marriage, August 2010 
3147 Jarko Fidrmuc, Time-Varying Exchange Rate Basket in China from 2005 to 2009, August 2010

3148 Ilja Neustadt and Peter Zweifel, Is the Welfare State Sustainable? Experimental Evidence on Citizens' Preferences for Redistribution, August 2010

3149 Marcus Dittrich and Andreas Knabe, Wage and Employment Effects of Non-Binding Minimum Wages, August 2010

3150 Shutao Cao, Enchuan Shao and Pedro Silos, Fixed-Term and Permanent Employment Contracts: Theory and Evidence, August 2010

3151 Ludger Woessmann, Cross-Country Evidence on Teacher Performance Pay, August 2010

3152 Lorenzo C. G. Pozzi, Casper G. de Vries and Jorn Zenhorst, World Equity Premium Based Risk Aversion Estimates, August 2010

3153 Volker Grossmann, Thomas M. Steger and Timo Trimborn, Dynamically Optimal R\&D Subsidization, August 2010

3154 Alexander Haupt, Tim Krieger and Thomas Lange, A Note on Brain Gain and Brain Drain: Permanent Migration and Education Policy, August 2010

3155 António Afonso and Christophe Rault, Long-run Determinants of Sovereign Yields, August 2010

3156 Franziska Tausch, Jan Potters and Arno Riedl, Preferences for Redistribution and Pensions. What can we Learn from Experiments?, August 2010

3157 Martin Kolmar and Andreas Wagener, Inefficient Group Organization as Optimal Adaption to Dominant Environments, August 2010

3158 Kai Carstensen, Klaus Wohlrabe and Christina Ziegler, Predictive Ability of Business Cycle Indicators under Test: A Case Study for the Euro Area Industrial Production, August 2010

3159 Horst Rottmann and Timo Wollmershäuser, A Micro Data Approach to the Identification of Credit Crunches, August 2010

3160 Philip E. Graves, Appropriate Fiscal Policy over the Business Cycle: Proper Stimulus Policies Can Work, August 2010

3161 Michael Binder and Marcel Bluhm, On the Conditional Effects of IMF Program Participation on Output Growth, August 2010

3162 Michael Binder, Qianying Chen, and Xuan Zhang, On the Effects of Monetary Policy Shocks on Exchange Rates, August 2010

3163 Felix J. Bierbrauer, On the Optimality of Optimal Income Taxation, August 2010 
3164 Nikolaus Wolf, Europe's Great Depression - Coordination Failure after the First World War, September 2010

3165 Dan Kovenock and Brian Roberson, Conflicts with Multiple Battlefields, September 2010

3166 Jean-Pierre Ponssard and Catherine Thomas, Capacity Investment under Demand Uncertainty. An Empirical Study of the US Cement Industry, 1994-2006, September 2010

3167 Jørgen Juel Andersen, Jon H. Fiva and Gisle James Natvik, Voting when the Stakes are High, September 2010

3168 Michael Hoel, Is there a Green Paradox?, September 2010

3169 Scott Alan Carson, Nineteenth Century US African-American and White Female Statures: Insight from US Prison Records, September 2010

3170 Gil S. Epstein, Yosef Mealem and Shmuel Nitzan, Political Culture and Discrimination in Contests, September 2010

3171 Sara Fisher Ellison, Jeffrey Greenbaum and Wallace P. Mullin, Diversity, Social Goods Provision, and Performance in the Firm, September 2010

3172 Silvia Dominguez-Martinez, Randolph Sloof and Ferdinand von Siemens, Monitoring your Friends, not your Foes: Strategic Ignorance and the Delegation of Real Authority, September 2010

3173 Marcus Dittrich and Beate Schirwitz, Union Membership and Employment Dynamics: A Note, September 2010

3174 Francesco Daveri, Paolo Manasse and Danila Serra, The Twin Effects of Globalization - Evidence from a Sample of Indian Manufacturing Firms, September 2010

3175 Florian Blöchl, Fabian J. Theis, Fernando Vega-Redondo and Eric O’N. Fisher, Which Sectors of a Modern Economy are most Central?, September 2010

3176 Dag Morten Dalen, Marilena Locatelli and Steinar Strøm, Longitudinal Analysis of Generic Substitution, September 2010

3177 Armin Falk, Stephan Meier and Christian Zehnder, Did we Overestimate the Role of Social Preferences? The Case of Self-Selected Student Samples, September 2010

3178 Christian Fahrholz and Cezary Wójcik, The Bail-Out! Positive Political Economics of Greek-type Crises in the EMU, September 2010

3179 Klaus Abberger and Wolfgang Nierhaus, The Ifo Business Cycle Clock: Circular Correlation with the Real GDP, September 2010 
3180 Walter Krämer and Gerhard Arminger, "True Believers" or Numerical Terrorism at the Nuclear Power Plant, September 2010

3181 Bernard M.S. Van Praag, Dmitri Romanov and Ada Ferrer-i-Carbonell, Happiness and Financial Satisfaction in Israel. Effects of Religiosity, Ethnicity, and War, September 2010

3182 Dimitrios Koumparoulis and Paul De Grauwe, Public Capital, Employment and Productivity: An Empirical Investigation for Greece, September 2010

3183 John Whalley and Tanmaya Shekhar, The Rapidly Deepening India-China Economic Relationship, September 2010

3184 Andreas Schäfer and Thomas Steger, History, Expectations, and Public Policy: Economic Development in Eastern Germany, September 2010

3185 Thomas Eichner and Marco Runkel, Subsidizing Renewable Energy under Capital Mobility, September 2010

3186 Konstantinos Angelopoulos and James Malley, Fear of Model Misspecification and the Robustness Premium, September 2010

3187 Philip E. Graves, A Note on the Design of Experiments Involving Public Goods, September 2010

3188 Glenn Ellison, How does the Market Use Citation Data? The Hirsch Index in Economics, September 2010

3189 Barbara Hanel and Regina T. Riphahn, The Employment of Mothers - Recent Developments and their Determinants in East and West Germany, September 2010

3190 Alexander Haupt and Silke Uebelmesser, Integration, Mobility, and Human Capital Formation, September 2010

3191 Vincenzo Galasso and Paola Profeta, When the State Mirrors the Family: The Design of Pension Systems, September 2010

3192 Stéphane Zuber and Geir B. Asheim, Justifying Social Discounting: The RankDiscounted Utilitarian Approach, September 2010 